\title{
UvWHI2 is Required for Stress Response and Pathogenicity in Ustilaginonidea Virens
}

\section{Shuai Meng}

China National Rice Research Institute

Jiehua Qiu

China National Rice Research Institute

Meng Xiong

China National Rice Research Institute

Zhiquan Liu

China National Rice Research Institute

Jane Sadhna Jagernath

China National Rice Research Institute

Huanbi Shi

China National Rice Research Institute

Yanjun Kou ( $\sim$ Kouyanjun241@hotmail.com )

China National Rice Research institute https://orcid.org/0000-0003-4189-0962

\section{Original article}

Keywords: Whi2, Rice false smut, Ustilaginoidea virens, Pathogenesis, Secondary spore

Posted Date: November 5th, 2020

DOl: https://doi.org/10.21203/rs.3.rs-101297/v1

License: (c) (1) This work is licensed under a Creative Commons Attribution 4.0 International License.

Read Full License 
$1 U v W H I 2$ is required for stress response and pathogenicity in Ustilaginonidea virens

2 Shuai Meng*, Jiehua Qiu*, Meng Xiong*, Zhiquan Liu, Jane Sadhna Jagernath, 3 Huanbin $\mathrm{Shi}^{\#}$, Yanjun $\mathrm{Kou}^{\#}$

4

5 State Key Laboratory of Rice Biology, China National Rice Research Institute, 6 Hangzhou. China, 311400 *Shuai Meng, Jiehua Qiu, Meng Xiong contributed equally to this work.

"For Correspondence: Huanbin Shi; Email: shihuanbin@caas.cn; Yanjun Kou; Email: kouyanjun241@hotmail.com

\section{Abstract:}

Background: Ustilaginoidea virens causes rice false smut disease, which emerges as a worldwide disease of rice. At present, some stress response related genes have been identified in $U$. virens, but it is not clear whether and how defects of stress responses affect the pathogenesis processes of $U$. virens. To answer this question, the function of a general stress response factor Whi2 was analyzed in $U$. virens.

Results: In this study, we identified UvWhi2 as a homolog of Saccharomyces cerevisiae Whi2 in $U$. virens. The relative expression level of UvWHI2 was significantly up-regulated during infection, suggesting that UvWhi2 may be involved in pathogenesis. Furthermore, knockout of UvWHI2 showed decreased the mycelial growth, increased in conidiation in the PS (potato sucrose) medium and a defect in pathogenicity. In addition, the RNA-Seq and phenotypic analysis showed that UvWhi2 is involved in response to oxidative, hyperosmotic, cell wall stresses, and nutrient limitation. Further studies revealed that the defects of stress responses of the $\Delta U v w h i 2$ mutant affected the formation of secondary spores on the nutrient limited surface and the rice surface, resulting in a significant reduction of pathogenicity of $U$. virens.

Conclusions: Our results suggest that UvWhi2 is necessary for fungal growth, stress responses, and the formation of secondary spores in $U$. virens. In addition, the defects 
of stress responses could affect the formation of secondary spores on the rice surface, and then compromise the pathogenicity of $U$. virens.

Keywords: Whi2, Rice false smut, Ustilaginoidea virens, Pathogenesis, Secondary spore

\section{Introduction}

Rice false smut disease is caused by the ascomycete filamentous fungus Ustilaginoidea virens, which has emerged to be one of the most devastating rice fungal diseases in the rice-cultivated areas (Fan et al., 2016, Liang et al., 2018, Guo et al., 2019, Qiu et al., 2019, Meng et al., 2020, Sun et al., 2020). The occurrence of rice false smut seriously affects high and stable yield and quality of rice (Lu et al., 2015, Zheng et al., 2017, Lin et al., 2018). During the booting period, the rice spikelets were infected by the $U$. virens, which hindered the nutrition transportation and the normal development of grains, resulting in the increase of empty chaff rate and the decrease of 1000-grain weight ( $\mathrm{Yu}$ et al., 2015, Tang et al., 2019). False smut balls contain various toxins, such as Ustilaginoidins and Ustiloxins, which could inhibit the assembly of microtubules in eukaryotic cells, disrupt cell mitosis and cause pathological changes in animal organs and plants (Lu et al., 2015, Zheng et al., 2016, Sun et al., 2017, Wang et al., 2017).

Thus far, only few pathogenesis-related proteins have been identified and characterized in $U$. virens, including the hypothetical protein UvPro1, the low-affinity iron transporter Uvt3277, two protein kinases UvPmk1 and UvCDC2, the cyclic adenosine monophosphate signaling proteins adenylate cyclase UvAc1 and phosphodiesterase UvPdeH, the Bax inhibitor UvBI-1, the effector Scre2 (Uv_1261), two transcriptional factors UvHox2 and UvCom1, the phosphatase UvPsr1, and the autophagy-related protein UvAtg8 (Lv et al., 2016, Zheng et al., 2017, Fang et al., 2019, Guo et al., 2019, Tang et al., 2019, Xie et al., 2019, Yu et al., 2019, Chen et al., 2020, 
Meng et al., 2020, Xiong et al., 2020). Among these, UvPmk1, UvCdc2, UvPro1, UvBI1, UvAc1, UvPdeh, UvPsr1, UvCom1, UvHox2, and UvAtg8 are involved in both various stresses responses and pathogenesis of $U$. virens. However, it is not clear whether and how defects of stress responses affect the pathogenesis processes of $U$. virens.

Whi2 (Whiskey2) is a general stress response factor. In yeast, ScWhi2 have been characterized to be involved in general stress response and coordinate nutrient status with cell cycle (Muller \& Reichert, 2011, Sadeh et al., 2011, Teng et al., 2018). Deletion of ScWHI2 causes decreased expression level of stress-associated genes, increased sensitivity to sodium ions, and disruption of the normal coordination of cell proliferation with nutrient availability (Kaida et al., 2002, Chen et al., 2018, Marsikova et al., 2020). In Podospora anserina, the Whi2 homolog has been shown to control nutrient sensing and the entrance of cells into the stationary phase and mycelial development (Timpano et al., 2016). In Colletotrichum orbiculare, CoWHI2 was reported to play key roles in regulation of transition from biotrophic infection to necrotrophic infection via regulation of TOR (target of rapamycin) signaling, which is involved in the coordination of cell growth and proliferation with the availability of growth factors and nutrients (Harata et al., 2016). Until now, the function of Whi2 remains largly unknown in various organisms.

In this study, UvWHI2, the homologous of S. cerevisiae WHI2 in U. virens was disrupted to characterize its function. RNA-Seq, qRT-PCR (quantitative Real-time PCR) and phenotypic analyses showed that UvWhi2 is involved in the growth, conidiation, pathogenicity, and various stresses response in $U$. virens. Furthermore, $\Delta U v w h i 2$ showed defects in the formation of secondary spores during germination on the nutrient limited rice surface, which highlights vital roles of UvWhi2 in stress response and pathogenicity in $U$. virens 


\section{Results}

Identification of UvWhi2 in $U$. virens

To identify the homolog of Whi2 in U. virens, we used ScWhi2 (accession number NP_014686) sequence as a query to do a blastP search in GeneBank to obtain the most closely match protein, which is named as UvWhi2 (accession number KDB15335.1) (Fig. 1a). Sequence analysis with motif scan revealed that UvWhi2 contained a signal peptide and GSDH (Glucose/Sorbosone dehydrogenase; Members of this family are glucose/sorbosone dehydrogenases that possess a beta-propeller fold.) domain. The phylogenetic analysis of the amino acid sequences of Whi2 from $U$. virens, Metarhizium robertsii, Aspergillus vadensis, Beauveria bassiana, Magnaporthe oryzae and $S$. cerevisiae, showed that Whi2 is conserved in various fungi, and UvWhi2 is highly similar to MrWhi2 (Fig. 1b). In addition, the qRT-PCR analysis showed that the transcription level of UvWHI2 at 3,5 and 9 dpi (days post inoculation) were significantly higher than mycelia (Fig. 1c), suggesting that UvWhi2 may have important roles in the infection process of $U$. virens.

101

\section{Knockout and complementation of $U v W H I 2$ in $U$. virens}

103 To analyze the function of $U v W H I 2$, the $U v W H I 2$ deletion mutants was generated by replacing the targeted gene with hygromycin resistant cassette in the wild type (WT) strain HWD-2 (Fig. 2a). Then, qRT-PCR and Southern blot assays were performed to confirm the targeted gene deletion events and exclude ectopic integrations (Fig. 2b). Six $\Delta U v w h i 2$ transformants were obtained with similar phenotypes, and two mutants ( $\Delta U v w h i 2-39$ and 43) were chosen for the subsequent further experiments (Fig. 2b). To

109 determine whether the altered phenotypes in the $\Delta U v w h i 2$ mutant were caused by

110 deletion of the $U v W H I 2$, the full-length gene copy of $U v W H I 2$ with its native promoter 111 was inserted into the vector $p F G L 823$ and transformed into the $\Delta U v w h i 2-39$ strain. The resultant $\Delta U v w h i 2-C$ strains were confirmed by PCR and qRT-PCR analyses, which 
113 showed that the abundance of $U v W H I 2$ transcript was comparable to that of the WT

114 strain (Fig. 2c, d). Furthermore, the colony morphology of $\Delta U v w h i 2-\mathrm{C}$ strain was the

115 same as that of the WT strain, suggesting that UvWHI2 functionally complemented the

116 phenotype of the $\Delta U v w h i 2$ strain.

117

118 Knockout of $\boldsymbol{U} \boldsymbol{v W H I} 2$ compromised fungal growth but enhanced conidiation 119 under nutrient-rich condition

120 To explore the function of UvWhi2 in vegetative growth and colony morphology of $U$.

121 virens, the growth of WT, $\Delta U v w h i 2$, and $\Delta U v w h i 2-\mathrm{C}$ strains were determined on the 122 PSA (potato sucrose agar) medium cultured for $14 \mathrm{~d}$. The $\Delta U v w h i 2$ mutant showed 123 smaller colonies than the WT and complementation strains (Fig. 3a). Considering the 124 reduction of colony size in the mutants, the dry weight of mycelium was measured after $1257 \mathrm{~d}$ of the PS liquid culture to determine whether there was a difference in biomass 126 between the WT and $\Delta U v w h i 2$ strains. The results showed that mycelial dry weight of 127 the $\Delta U v w h i 2$ strains were lower than that of the WT strain (Fig. 3c). In contrast, the 128 colony morphology and mycelia dry weight were rescued in the $\Delta U v w h i 2-\mathrm{C}$ strain (Fig. $1293 \mathrm{~b}, \mathrm{c})$, suggesting that UvWhi2 plays important roles in the vegetative growth in $U$. 130 virens. The conidia play an important role during the infection processes of $U$. virens (Fan 133 et al., 2016, Xie et al., 2019, Meng et al., 2020, Xiong et al., 2020). To analysis the 134 function of $U v W H I 2$ in conidiation, the WT, $\Delta U v w h i 2$ and $\Delta U v w h i 2-\mathrm{C}$ strains were 135 cultured in the liquid PS medium for $7 \mathrm{~d}$. Although the vegetative growth of $\Delta U v w h i 2$ 136 strain reduced, the conidial production of the $\Delta U v w h i 2$ mutant was more than those of 137 the WT and $\Delta U v w h i 2-\mathrm{C}$ strains (Fig. $3 \mathrm{~d}$, e). These results showed that UvWhi2 acts as 138 a negative regulator in the sporulation production process in nutrient rich medium. 
141 To evaluate whether deletion of $U v W H I 2$ can affect the virulence of rice false smut, the

142 WT, $\Delta U v w h i 2-39$ and 43, and $\Delta U v w h i 2-\mathrm{C}$ strains were inoculated into panicles of the 143 susceptible rice cultivar Wanxian 98 (Oryza sativa L. indica). Three weeks after the 144 inoculations, it was found that the number of smut balls formed in panicles inoculated 145 with the $\Delta U v w h i 2-39$ and 43 strains was significantly less than those of the WT and $146 \Delta U v w h i 2-\mathrm{C}$ strains (Fig. 4a, b). Therefore, knockout of $U v W H I 2$ remarkably attenuated $147 U$. virens virulence, suggesting that UvWhi2 is a key regulator factor of the 148 pathogenicity in $U$. virens.

The RNA-Seq and phenotypic analysis showed that UvWhi2 is involved in various stresses responses

152 To further explore the function of $U v W H I 2$ in $U$. virens, the RNA-Seq analysis was performed with the $\Delta U v w h i 2$ and WT strains. RNA-Seq analysis showed that the number of SDEGs (significantly differentially expressed gene, FDR adjusted $\mathrm{p} \leq 0.05$ $\&$ absolute $\log _{2}$ fold $\geq 2$ ) was 802 , of which 515 were up-regulated and 287 were downregulated in the $\Delta U v w h i 2$ mutant when compared with the WT strain (Table S1). And the SDEGs could be divided into three major functional groups: biological process, cellular component, and molecular function based on GO analysis (Fig. S1a). Notably, some peroxidase activity genes, chitin deposition genes, and peroxidase activity genes were shown in the differential expression genes (Fig. S1b).

Synthesis of the laccase, peroxidase, chitin synthase, and hyperosmotic genes play an important role in stress response (Martin et al., 2007, Song et al., 2010, Li et al., 2016, Zheng et al., 2016). Comparing with the WT strain, the expression level of chitin deposition genes ( $U v 8 b \_7958, U v 8 b \_7948, U v 8 b \_4757$, and $\left.U v 8 b \_3223\right)$ of the

$166 \Delta U v w h i 2$ strain were significantly lower (Fig. 5a), suggesting that UvWhi2 may be involved in the cell wall stresses response. Then, the WT and $\Delta U v w h i 2$ strains were 
white (CFW) and $120 \mu \mathrm{g} / \mathrm{mL}$ Congo red (CR), which are cell wall disruptors, to

170 determine the sensitivity of indicated strain to these agents. The results showed that the

$171 \Delta U v w h i 2$ strains were more sensitive to the SDS, CFW and CR than the WT strain (Fig.

$1725 \mathrm{~d}, \mathrm{e})$. In addition, the expression levels of laccase and peroxidase activity genes

173 (Uv8b_1784,Uv8b_990,Uv8b_6387,Uv8b_4892, and Uv8b_4252) were significantly

174 reduced in the $\Delta U v w h i 2$ strain (Fig. 5c). Moreover, the growth of both $\Delta U v w h i 2$ strains

175 was more strongly inhibited by the presence of $0.03 \% \mathrm{H}_{2} \mathrm{O}_{2}$ (Oxidative stress agent)

176 than that of the WT and $\Delta U v w h i 2-C$ strains (Fig. $5 \mathrm{~d}$, e). In contrast, the expression

177 levels of hyperosmotic activity genes ( $U v 8 b_{-} 1888$ and $\left.U v 8 b \_6309\right)$ were notably

178 increased compared with the WT strain (Fig. 5b). Consistently, in the presence of 0.4

$179 \mathrm{M} \mathrm{NaCl}$ or $0.7 \mathrm{M}$ sorbitol, the growth of the WT strain was reduced by $70 \%$, but that

180 of $\Delta U v w h i 2$ mutants reduced by approximate $35 \%$, indicating $\Delta U v w h i 2$ was less

181 sensitive to osmotic stresses than the WT strain (Fig. 5d, e). In conclusion, these results

182 suggested that $U v W H I 2$ contributes to the responses to cell wall, oxidative and osmotic

183 stresses in $U$. virens.

184

In addition, the RNA-Seq and qRT-PCR results also showed that the expression 186 levels of sugar-related genes (Uv8b_1668 and $\left.U v 8 b_{-} 1099\right)$ in the $\Delta U v w h i 2$ mutant

187 strain were lower than the WT strain (Fig. 6a). We assumed that UvWhi2 may be 188 involved in the regulation of cell growth and proliferation in response to nutrient stress 189 as Whi2 in other fungus (Kaida et al., 2002, Leadsham et al., 2009, Chen et al., 2018).

190 To confirm this hypothesis, the WT, $\Delta U v w h i 2$, and $\Delta U v w h i 2-\mathrm{C}$ strains were cultured 191 on the SD (synthetic dropout medium), SD-G (synthetic dropout medium without 192 glucose), and SD-N (synthetic dropout medium without nitrogen) plates. After $15 \mathrm{~d}$ of 193 incubation, comparing with the WT strain on the SD medium, the $\Delta U v w h i 2$ strains were 194 significantly reduced in mycelia growth on both the SD-G and SD-N plates (Fig. 6b, c).

195 In contrast, the complementation strain $\Delta U v w h i 2-\mathrm{C}$ showed similar phenotypes as the 196 WT strain under all observed stress conditions. All these results indicated that UvWhi2 
197 is involved in the regulation of nutrient stresses responses in $U$. virens.

199 Defects in the stress response compromised the secondary spore formation on the rice surface in the $\Delta U v w h i 2$ mutant

201 In order to further determine whether the defects in stress response in the $\Delta U v w h i 2$ 202 mutant affect pathogenesis, we carefully observed the germination processes. We found that the $\Delta U v w h i 2$ mutant had a significant increase in the formation of secondary spores

204 on the PSA medium plate (Fig. 7a). In contrast, when the conidia were germinated on 205 the agarose plate without other nutrient supplementation, the formation of secondary 206 spores was sharply decreased (Fig. 7b). Moreover, secondary spore formation of the $207 \Delta U v w h i 2$ mutant was even rarer on the rice surface (Fig. 7c), which might have limited 208 nutrient and oxidative stress (Fan et al., 2014, Wang et al., 2019). These results 209 suggested that the secondary spore formation of the $\Delta U v w h i 2$ mutant is compromised 210 under the nutrient limited condition and rice surface. And we inferred that the highly 211 decreased secondary spore formation on the rice surface is one of the main reasons for 212 the lost pathogenicity in the $\Delta U v w h i 2$ mutant.

\section{Discussion}

215 Rice false smut has posed a serious threat to the yield and quality of rice in recent years, 216 so it is important to study the pathogenesis of $U$. virens. Here, we identified a general 217 stress response factor Whi2 in U. virens. According to our results, UvWhi2 play 218 important roles in the hyphal growth, conidiation, various stresses responses, and 219 pathogenicity.

In $U$. virens, a series of genes related to pathogenicity have been identified, but the 222 pathogenic mechanism of this fungus is still largely unknown (Zheng et al., 2016, 223 Zheng et al., 2017, Yu et al., 2019, Xiong et al., 2020, Lv et al., 2016, Tang et al., 2019, 224 Xie et al., 2019). In this study, deletion of UvWHI2 highly reduced the pathogenicity of 
$U$. virens to rice. Through a series of analysis, we found that, in addition to the

226 compromised fungal growth, the reduced pathogenicity in $\Delta U v w h i 2$ mutant was likely 227 caused by highly reduced formation of secondary spores on the rice surface. During 228 pathogenic process of $U$. virens, the formation of secondary spores tends to greatly 229 increase the amount of inoculums available to infect rice plants (Fan et al., 2014). In 230 addition, the deletion mutant of $U v A T G 8$, which encodes an ubiquitin-like protein 231 required for autophagy-independent function, has defects in formation of secondary 232 spores and showed the loss of pathogenicity, suggesting that the formation of secondary 233 spores play an important role in the infection of $U$. virens (Meng et al., 2020). In the $234 \Delta U v w h i 2$ mutant, secondary spore formation of the $\Delta U v w h i 2$ mutant was rarer on the 235 rice surface. Thus, we inferred that the main reason for the highly reduced of 236 pathogenicity of the $\Delta U v w h i 2$ mutant may be the decrease of secondary spore formation.

In U. virens, UvPmk1, UvCdc2, UvPro1, UvBI-1, UvAc1, UvPdeh, UvPsr1, UvCom1, UvHox2, and UvAtg8 are involved in both various stresses responses and 240 pathogenesis (Lv et al., 2016, Guo et al., 2019, Tang et al., 2019, Xie et al., 2019, Yu et 241 al., 2019, Meng et al., 2020, Xiong et al., 2020). However, it is not clear whether and 242 how defects of stress responses affect the pathogenesis processes of $U$. virens. This 243 study analyzed the role of a general stress response factor Whi2 to reveal the 244 relationship between the stress response and pathogenicity in U. virens. In yeast, the 245 homolog of Whi2, ScWhi2, has been identified to be involved in various stress 246 responses (Mendl et al., 2011, Harata et al., 2016, Teng et al., 2018). ScWhi2 interacts 247 with the protein ScPsrl to activate STRE-mediated genes, possibly via the 248 dephosphorylation of the Msn2 transcription factor (Kaida et al., 2002, Boeckstaens et 249 al., 2014), and then regulates the downstream stress response. In P. anserine, PaWhi2 250 is also likely affected in nutrient sensing and regulate the vegetative growth (Timpano 251 et al., 2016). In this study, the results of comparative RNA-Seq analysis showed that some stress response genes were differential expressed in the WT and $\Delta U v w h i 2$ mutant 
strains. Consistent with the RNA-Seq results, knockout of UvWHI2 reduced the sensitive to salinity stress, increased the sensitive to oxidative stress and cell wall stresses, and decreased the hyphal growth under nutrient limited conditions, indicating 256 that UvWhi2 is also involved in various stresses responses in U. virens. Furthermore, 257 we noted that deletion of $U v W H I 2$ enhanced secondary spore formation on the PSA 258 plate, on the contrary, reduced secondary spore formation on the nutrient limited surface, 259 including the agarose plate without other nutrient supplementation and the rice surface.

260 These results suggest that the defects of stress responses in the $\Delta U v w h i 2$ mutant 261 affected the formation of secondary spores on the nutrient limited rice surface, leading 262 to a severe reduction in pathogenicity of $U$. virens.

In conclusion, our results suggest that UvWhi2 is necessary for fungal growth, stress responses, conidiation, secondary spore formation, and pathogenicity in $U$. virens. In addition, the defects of stress responses could affect the formation of secondary spores on the rice surface, and then affect the pathogenicity of $U$. virens.

\section{Materials and methods}

\section{Sequence analysis}

271 The sequences of the genes and proteins used in this study were downloaded from the

272 National Center for Biotechnology Information (NCBI, https://www.ncbi.nlm.nih.gov/).

273 The motif scan was performed online (https://myhits.isb-sib.ch/cgi-bin/motif_scan).

274 The protein sequence alignments were processed using EPSript 3.0. The following 275 phylogenetic analyses were performed using MEGA 5.0 with a neighbor-joining 276 algorithm method (Robert \& Gouet, 2014).

\section{Fungal growth}

279 The $U$. virens wild-type strain HWD-2 was a kind gift from the Huazhong agriculture 280 university (Hubei, Wuhan). The U. virens strain was cultured at a constant temperature 
281 of $28^{\circ} \mathrm{C}$ on the PSA (potato $200 \mathrm{~g} / \mathrm{L}$, sucrose $20 \mathrm{~g} / \mathrm{L}$, and agar $20 \mathrm{~g} / \mathrm{L}$ ). For liquid culture, 282 the mycelia were shaken in the PS (200 g/L potato and $20.0 \mathrm{~g} / \mathrm{L}$ sucrose $)$ medium at $283170 \mathrm{rpm}$ and $28^{\circ} \mathrm{C}$ for $7 \mathrm{~d}$. The mycelia growth, mycelia dry weight, conidia 284 concentration was measured as described (Meng et al., 2020). The conidial germination 285 experiments were performed on the PSA, agarose plates, and the rice sheath 286 respectively. Three independent biological experiments were performed with three replicates each time.

\section{Construction of the $\Delta U v w h i 2$ strains and complementation analyses}

290 To construct the UvWHI2 deletion mutant strain using the gene replacement strategy, 291 the 1026 bp upstream and 989 bp downstream flanking sequences of UvWHI2 were 292 amplified from the genomic DNA of the HWD-2 strain using the primer pairs Uvwhi2$2935 \mathrm{~F} / \mathrm{R}$ and Uvwhi2-3F/R (Table S2). Then, these flanking fragments of $U v W H I 2$ were 294 cloned into vector $p F G L 821$ (Addgene: 58224, www.addgene.org) (Xiong et al., 2020).

295 For the complementation, the UvWHI2 fragment, including coding region, promoter 296 and 3'-UTR regions of UvWHI2 (Table S2), was inserted into the vector pFGL823, 297 which was derived from the replacement of hygromycin phosphotransferase gene 298 cassette in pFGL821 by neomycin resistance gene. Agrobacterium-mediated 299 transformation was applied for genetic transformation in $U$. virens following the 300 protocol (Yu et al., 2015). The correct transformants of $\Delta U v w h i 2$ and complementation 301 assay were ascertained using Southern blot and qRT-PCR analyses.

\section{Southern blotting, RNA isolation and qRT-PCR analyses}

304 For Southern blot analysis, the genomic DNA of the WT strain and mutants were 305 extracted and digested with Xho I. Then the digested products of genomic DNA were 306 size fractionated through $0.8 \%$ agarose gel and mounted onto positively charged nylon 307 membrane (GE Healthcare, Buckinghamshire, UK). The purified probe UvWHI2308 probe-F/R (Table S2) was DIG-labeled with Labeling Reagents (GE Healthcare, 
309 Buckinghamshire, UK) to hybridize with the digested products of the WT and $\Delta U v w h i 2$ 310 strains. All the hybridization process was executed following the manufacturing 311 instruction of Amersham ${ }^{\mathrm{TM}}$ AlkPhos Direct Labeling Reagents (GE Healthcare, 312 Buckinghamshire, UK). Then, ChemiDoc XRS+ system (Bio-Rad, Hercules, USA) was 313 used to detect the signals of Southern Blotting.

The total RNA of the fungal mycelia was extracted using the Fungal RNA Kit 200

316 (OMEGA). The first-stranded cDNA was synthesized with a reverse transcription kit 317 (TaKaRa, Japan), and then TB Green ${ }^{\mathrm{TM}}$ Premix Ex Taq ${ }^{\mathrm{TM}}$ (Tli RnaseH Plus) was used 318 for qRT-PCR analysis. The $\beta$-Tubulin gene was used as the endogenous reference gene 319 (Table S2). The relative expression levels of UvWHI2 gene were calculated using the $2^{-\Delta \Delta} \mathrm{Ct}$ method.

\section{Pathogenicity assay}

323 For the pathogenicity assay, liquid culture of mycelia and conidia $\left(1 \times 10^{6}\right.$ conidia $\left./ \mathrm{mL}\right)$ 324 were mixed together, and broken down in a juice blender. Then, the conidia suspension was injected into the panicles of selected rice cultivar Wanxian 98 (a susceptible rice

326 cultivar, Oryza sativa L. indica) at the booting stage. The inoculated plants were 327 cultivated under $12 \mathrm{~h}$ light/dark conditions at $25^{\circ} \mathrm{C}$ and $95 \%$ humidity. Then, the 328 phenotype of smut balls was measured and scanned at $21 \mathrm{dpi}$. All the experiments in 329 this part were repeated three times in each test.

\section{RNA-Seq library preparation and Illumina sequencing}

332 Total RNA was extracted from seven-day-old mycelia and conidia of WT and $\Delta U v w h i 2-$ 33339 strains using the Fungal RNA Kit 200 (OMEGA). A total of $3 \mu \mathrm{g}$ RNA and the 334 NEBNext ${ }^{\circledR}$ UltraTM RNA Library Prep Kit was used to generate RNA-Seq 335 transcriptome libraries for sequencing on Illumina ${ }^{\circledR}$ (NEB, USA) following 336 manufacturer's recommendations. 

quality reads were removed to obtain clean reads base on their error rate, Q20, Q30,

340 and GC contents. Reference genome and gene model annotation files were downloaded 341 from genome website directly 342 (https://ftp.ncbi.nlm.nih.gov/genomes/all/GCA/000/687/475/GCA_000687475.1_Ass 343 embly_for_version_1_of_the_Villosiclava_virens_genome/). Then, the eatureCounts 344 v1.5.0-p3 was used to map the clean reads to reference genome. And each gene 345 expression level was based on the FPKM (Fragments Per Kilobase per Million). 346 Differential expression genes of two samples was obtained using the DESeq2 software 347 with FDR adjusted $\mathrm{p} \leqslant 0.05 \&$ absolute $\log 2$ fold $\geqslant 2$. Gene Ontology (GO) 348 enrichment analysis was performed under the Bonferroni-corrected P-value $\leqslant 0.05$ 349 compared with the whole-transcriptome background. GO functional enrichment 350 analysis was finished on the website (https://www.omicshare.com/tools/).

\section{Abiotic stresses response analysis}

352 To test the sensitivity to various abiotic stresses, the WT, $\Delta U v w h i 2$, and $\Delta U v w h i 2-\mathrm{C}$ 353 strains were cultured at $28^{\circ} \mathrm{C}$ for $15 \mathrm{~d}$ on the PSA and PSA with $0.03 \% \mathrm{H}_{2} \mathrm{O}_{2}, 0.4 \mathrm{M}$ $354 \mathrm{NaCl}$ (Solarbio, S8210), $0.7 \mathrm{M}$ sorbitol (Coolaber, CS10381), 0.03\% SDS (Sigma, 355 L3771), $120 \mu \mathrm{g} / \mathrm{mL}$ CFW (Sigma, F3543), and $120 \mu \mathrm{g} / \mathrm{mL}$ CR (Solarbio, IC1000) to 356 measure the colony diameters. The formula of inhibition rate was calculated as follow: 357 Inhibition rate $=($ average of strain colony diameters on the PSA - the average of strain 358 colony diameters on the PSA with different chemicals)/average of the strain colony 359 diameters on the PSA $\times 100 \%$. For the nutrient starvation assay, the SD (Yeast nitrogen 360 base without amino acids $1.7 \mathrm{~g} / \mathrm{L}$, Ammonium sulfate, $5 \mathrm{~g} / \mathrm{L}$ glucose 20g/L, and agar 20 $361 \mathrm{~g} / \mathrm{L}$ ) medium, SD-G (Yeast nitrogen base without amino acids $1.7 \mathrm{~g} / \mathrm{L}$, Ammonium 362 sulfate, $5 \mathrm{~g} / \mathrm{L}$ glucose $20 \mathrm{~g} / \mathrm{L}$, and agar $20 \mathrm{~g} / \mathrm{L}$ ), and SD-N (Yeast nitrogen base without 363 amino acids $1.7 \mathrm{~g} / \mathrm{L}$, glucose $20 \mathrm{~g} / \mathrm{L}$, and agar $20 \mathrm{~g} / \mathrm{L}$ ) plates were used. All the experiments were performed three times with three replicates. 


\section{Abbreviations}

367 Whi2, Whiskey2; CFW, calcofluor white; CR, congo red; d, days; dpi, days post 368 inoculation; Kb, kilobases; F. graminearum, Fusarium graminearum; M. oryzae,

369 Magnaporthe oryzae; mins, minutes; PS, potato sucrose medium; PSA, potato sucrose 370 agar medium; SD, synthetic dropout medium; SD-G, synthetic dropout medium without 371 glucose; SD-N, synthetic dropout medium without nitrogen; S. cerevisiae, 372 Saccharomyces cerevisiae; SDS, Sodium dodecyl sulfate; U. virens, Ustilaginoidea 373 virens; P. anserina, Podospora anserina; WT, wild type; qRT-PCR, quantitative real374 time polymerase chain reaction.

\section{Consent for publication}

377 Not applicable.

Ethics approval and consent to participate

380 Not applicable.

\section{Availability of data and materials}

383 The datasets supporting the conclusions of this article are included within the article 384 and its additional files.

\section{Competing interests}

387 There is no conflict of interest.

389 Funding: This research was funded by Zhejiang Provincial Natural Science Foundation

390 of China, grant number "LQ19C140004" and key R\&D project of Zhejiang Province, 391 grant number "2019C02018". 
394 YJK and HBS planned and designed the research. SM, JHQ, MX, JSJ, and HBS 395 performed experiments, conducted field work, and analyzed data etc. SM, MX, and 396 YJK wrote the manuscript. All authors read, revised and approved the final manuscript.

\section{Acknowledgements}

399 This project was supported by the Chinese Academy of Agricultural Sciences under the 400 "Elite Youth" program, the Agricultural Sciences and Technologies Innovation Program. 401 We thank Prof. Junbin Huang for providing the HWD-2 strain.

\section{Supplemental information}

Table S1. Differently expression genes and GO enrichment analysis between the WT and $\Delta U v w h i 2$ strains.

Table S2. Primers used in this study.

Fig. S1 Comparative transcriptomic analysis of the WT and $\Delta U v w h i 2$ strains.

Fig. 1 Identification of UvWhi2 in Ustilaginoidea virens. a, The multiple alignment of amino acid sequences of UvWhi2, MrWhi2, BbWhi2, MoWhi2, AvWhi2 and

411 ScWhi2. Amino acids in black and gray represent amino acids identity and fifty percent 412 similarity, respectively. b, Phylogeny analysis of Whi2 orthologs from different fungi 413 was constructed by MEGA 5.0 using the neighbor-joining method. The sequences 414 included UvWhi2 (U. virens, KDB15335.1), MrWhi2 (Metarhizium robertsii, 415 XM_007820024.1), BbWhi2 (Beauveria bassiana, XM_008598486.1), MoWhi2 416 (Magnaporthe oryzae, XP_003714147.1), AvWhi2 (Aspergillus vadensis, 417 XM_025709355.1), and ScWhi2 (Saccharomyces cerevisiae, NP_014686). c, The 418 transcriptional analysis of UvWHI2 in the mycelia and inoculated panicles of WanXian 41998 (oryza sativa L. indica) at 3, 5, and 9 dpi. The data represents the mean \pm SD from 420 three independent replicates. The data were subject to Duncan's Test and the significant 
differences were indicated in the figure with three asterisks $(* * *, p<0.001)$.

Fig. 2 Targeted gene deletion of $U v W H I 2$ and complementation assay in $U$, virens.

a, Construction strategy for the $U v W H I 2$ gene deletion mutant in $U$. virens. The coding region of UvWHI2 was replaced with the hygromycin phosphotransferase gene cassette $(H Y G)$ by homologous recombination. b, The Southern blot assay was used to validate the loss of $U v W H I 2$ in the $\Delta U v w h i 2$ deletion mutant. The Xho I enzyme was used to digested genomic DNA of the WT and $\Delta U v w h i 2$ strains. The digested genomic were processed for the Southern blotting with the $1 \mathrm{~Kb}$ downstream of $U v W H I 2$ as probe. c, qRT-PCR analysis of the expression level of UvWHI2 in the WT, $\Delta U v w h i 2$, and $\Delta U v w h i 2-\mathrm{C}$ strains. The data represents the mean $\pm \mathrm{SD}$ from three independent replicates. The asterisks $(* * *)$ indicate a $\mathrm{P}$ value $<0.001$.

Fig. 3 Deletion of $U v W H I 2$ results in decreased vegetative growth and increased conidiation in $\boldsymbol{U}$. virens. a, Mycelia growth of the WT, $\Delta U v w h i 2$, and $\Delta U v w h i 2-\mathrm{C}$ strains on the PSA (potato sucrose agar) medium in dark at $28^{\circ} \mathrm{C}$ for $15 \mathrm{~d}$. b, Colony morphology of indicated strains. c, Dry weight of mycelia was measured in the PS medium after $7 \mathrm{~d}$ culture. $\mathrm{d}$ and e, Knockout of $U v$ WHI2 enhanced conidiation under nutrient-rich condition. Data represents the mean \pm SD from three independent replicates. The data were subject to Duncan's Test and the significant differences were indicated in the figure with three asterisks $(* * *, p<0.001)$. Scale bar $=5 \mu \mathrm{m}$.

Fig. 4 UvWhi2 is required for pathogenesis in $\boldsymbol{U}$. virens. a, Disease symptoms of 444 indicated strain on the rice panicles of WanXian 98 at 21 dpi. b, Statistical analysis of 445 the average number of false smut balls on the inoculated spikelets. Each experiment 446 was performed with three independent biological experiments and more than thirty 447 panicles were inoculated each time. Data were showed as Mean $\pm \operatorname{SD}(n=3)$. ***, $\mathrm{p}<$ $448 \quad 0.001$. 
450 Fig. 5 UvWHI2 contribute to the stress responses to the cell wall, oxidative and 451 osmotic agent in $\boldsymbol{U}$. virens. a, b and c, qRT-PCR analysis of the expression of the genes 452 related to laccase, peroxidase, chitin deposition and hyperosmotic activities, 453 respectively. $\mathrm{d}$, The tested strains grown on the PSA or PSA with $0.03 \% \mathrm{H}_{2} \mathrm{O}_{2}$ 454 (Oxidative stress agent), 0.03\% SDS (Sodium dodecyl sulfate), $120 \mu \mathrm{g} / \mathrm{mL}$ CFW 455 (Calcofluor white), $120 \mu \mathrm{g} / \mathrm{mL} \mathrm{CR}$ (Congo red), $0.4 \mathrm{M} \mathrm{NaCl}$, or $0.7 \mathrm{M}$ sorbitol. Typical 456 cultures were photographed after $15 \mathrm{~d}$ at $28^{\circ} \mathrm{C}$. e, Statistical analysis of inhibition rate 457 of tested strains with different stress agents. The diameters of colonies were measured 458 and calculated. Similar results were obtained by three repeated experiments. The error 459 bars represent the standard deviation and the asterisk represents the significant 460 difference compared to the WT strain under the same conditions $\left(^{*}, \mathrm{p}<0.01 ; * *, \mathrm{p}<\right.$ $461 \quad 0.005 ; * * *, \mathrm{p}<0.001)$.

Fig. $6 \mathrm{UvWhi} 2$ is involved in the regulation of nutrient stresses responses in $U$. virens. a, qRT-PCR analysis of the genes related to sugar synthesis. b, The growth of tested strains on the SD (synthetic dropout medium), SD-G (synthetic dropout medium without glucose), and SD-N (synthetic dropout medium without nitrogen) medium.

467 Typical cultures were photographed after culturing for $15 \mathrm{~d}$ at $28^{\circ} \mathrm{C}$. The diameters of colonies were measured to calculate the inhibition rate. c, Statistical analysis of tested strains grown on the SD-G and SD-N medium at $15 \mathrm{~d}$. Similar results were obtained by three repeated experiments. $* *$ or $* * *, p<0.005$ or $\mathrm{p}<0.001$.

473 and rice sheath surface. a-c, Conidial germination of $U$. virens on the PSA and agarose 474 plates and the rice sheath. The conidia were inoculated on the indicated surface at $28^{\circ} \mathrm{C}$ 475 for $3 \mathrm{~d}$. Scale bar $=3 \mu \mathrm{m}$. 
Boeckstaens M, Llinares E, Van Vooren P, Marini A, M, (2014). The TORC1 effector kinase Npr1 fine tunes the inherent activity of the Mep2 ammonium transport protein. Nat Commun 5: 3101. https://doi.org/10.1038/ncomms4101

Chen X, Wang G, Zhang Y, Dayhoff-Brannigan M, Diny NL, Zhao M et al, (2018). Whi2 is a conserved negative regulator of TORC1 in response to low amino acids. PLoS Genet 14, (8): 1007592. https://doi.org/10.1371/journal.pgen.1007592

Chen XY, Hai D, Tang JT, Liu H, Huang JB, Luo CX et al, (2020). UvCom1 is an important regulator required for development and infection in the rice false smut fungus Ustilaginoidea virens. Phytopathology 110, (2): 483-93. https://doi.org/10.1094/PHYTO-05-19-0179-R

Fan J, Guo XY, Huang F, Li Y, Liu YF, Li L et al, (2014). Epiphytic colonization of Ustilaginoidea virens on biotic and abiotic surfaces implies the widespread presence of primary inoculum for rice false smut disease. Plant Pathology 63: 937-45. https://doi.org/10.1111/ppa.12167

Fan J, Yang J, Wang YQ, Li GB, Li Y, Huang F et al, (2016). Current understanding on Villosiclava virens, a unique flower-infecting fungus causing rice false smut disease. Mol Plant Pathol 17, (9): 1321-30. https://doi.org/10.1111/mpp.12362

Fang A, Gao H, Zhang N, Zheng X, Qiu S, Li Y et al, (2019). A novel effector gene SCRE2 contributes to full virulence of Ustilaginoidea virens to rice. Front Microbiol 10: 845. https://doi.org/10.3389/fmicb.2019.00845

Guo W, Gao Y, Yu Z, Xiao Y, Zhang Z, Zhang H, (2019). The adenylate cyclase UvAc1 and phosphodiesterase UvPdeH control the intracellular cAMP level, development, and pathogenicity of the rice false smut fungus Ustilaginoidea virens. Fungal Genet Biol 129: 65-73. https://doi.org/10.1016/j.fgb.2019.04.017

Harata K, Nishiuchi T, Kubo Y, (2016). Colletotrichum orbiculare WHI2, a yeast stressresponse regulator homolog, controls the biotrophic stage of hemibiotrophic infection through TOR signaling. Mol Plant Microbe Interact 29, (6): 468-83. https://doi.org/10.1094/MPMI-02-16-0030-R

Kaida D, Yashiroda H, Tohe A, Kikuchi Y, (2002). Yeast Whi2 and Psr1-phosphatase form a complex and regulate STRE-mediated gene expression. Genes Cells 7, (6): 543-52. https://doi.org/10.1046/j.1365-2443.2002.00538.x

Leadsham JE, Miller K, Ayscough KR, Colombo S, Martegani E, Sudbery P et al, (2009). Whi2p links nutritional sensing to actin-dependent Ras-cAMP-PKA regulation and apoptosis in yeast. $J$ Cell Sci 122, (Pt 5): 706-15. https://doi.org/10.1242/jcs.042424

Li M, Liu X, Liu Z, Sun Y, Liu M, Wang X et al, (2016). Glycoside hydrolase MoGls2 controls asexual/sexual development, cell wall integrity and infectious growth in the rice blast fungus. PLoS One 11, (9): e0162243. https://doi.org/10.1371/journal.pone.0162243

Liang Y, Han Y, Wang C, Jiang C, Xu JR, (2018). Targeted deletion of the USTA and 
UvSLT2 genes efficiently in Ustilaginoidea virens with the CRISPR-Cas9 system. Front Plant Sci 9: 699. 10.3389/fpls.2018.00699

Lin X, Bian Y, Mou R, Cao Z, Cao Z, Zhu Z et al, (2018). Isolation, identification, and characterization of Ustilaginoidea virens from rice false smut balls with high ustilotoxin production potential. $J$ Basic Microbiol 58, (8): 670-8. https://doi.org/10.1002/jobm.201800167

Lu S, Sun W, Meng J, Wang A, Wang X, Tian J et al, (2015). Bioactive bis-naphthogamma-pyrones from rice false smut pathogen Ustilaginoidea virens. J Agric Food Chem 63, (13): 3501-8. https://doi.org/10.1021/acs.jafc.5b00694

Lv B, Zheng L, Liu H, Tang J, Hsiang T, Huang J, (2016). Use of random T-DNA mutagenesis in identification of gene $U v P R O 1$, a regulator of conidiation, stress response, and virulence in Ustilaginoidea virens. Front Microbiol 7: 2086. https://doi.org/10.3389/fmicb.2016.02086

Marsikova J, Pavlickova M, Wilkinson D, Vachova L, Hlavacek O, Hatakova L et al, (2020). The Whi2p-Psr1p/Psr2p complex regulates interference competition and expansion of cells with competitive advantage in yeast colonies. Proc Natl Acad Sci U S A 117, (26): 15123-31. https://doi.org/10.1073/pnas.1922076117

Martin JE, Zheng YW, Mark AJ, Nicholas Smirnoff, Nicholas JT, (2007). Generation of reactive oxygen species by fungal NADPH oxidases is required for rice blast disease. Proc Natl Acad, $U \quad S \quad A$ 104, (28): 11772-7. https://doi.org/10.1073/pnas.0700574104

Mendl N, Occhipinti A, Muller M, Wild P, Dikic I, Reichert AS, (2011). Mitophagy in yeast is independent of mitochondrial fission and requires the stress response gene WHI2. Journal of Cell Science 124, (8): 1339-50. https://doi.org/10.1242/jcs.076406

Meng S, Xiong M, Jane SJ, Wang CC, Qiu JH, Shi HB et al, (2020). UvAtg8-mediated autophagy regulates fungal growth, stress responses, conidiation, and pathogenesis in Ustilaginoidea virens. Rice 13: 56-69. https://doi.org/10.1186/s12284-020-00418-z

Muller M, Reichert AS, (2011). Mitophagy, mitochondrial dynamics and the general stress response in yeast. Biochem Soc Trans 39, (5): 1514-9. https://doi.org/10.1042/BST0391514

Qiu JH, Meng S, Deng YZ, Huang SW, Kou YJ, (2019). Ustilaginoidea virens: a fungus infects rice flower and threats world rice production. Rice Science 26, (4): 199206. https://doi.org/10.1016/j.rsci.2018.10.007

Robert X, Gouet P, (2014). Deciphering key features in protein structures with the new ENDscript server. Nucleic Acids Res 42, (Web Server issue): W320-4. https://doi.org/10.1093/nar/gku316

Sadeh A, Movshovich N, Volokh M, Gheber L, Aharoni A, (2011). Fine-tuning of the Msn2/4-mediated yeast stress responses as revealed by systematic deletion of Msn2/4 partners. Mol Biol Cell 22, (17): 3127-38. https://doi.org/10.1091/mbc.E10-12-1007 
Song W, Dou X, Qi Z, Wang Q, Zhang X, Zhang H et al, (2010). R-SNARE homolog MoSec22 is required for conidiogenesis, cell wall integrity, and pathogenesis of Magnaporthe oryzae. PLoS One 5, (10): e13193. https://doi.org/10.1371/journal.pone.0013193

Sun W, Fan J, Fang A, Li Y, Tariqjaveed M, Li D et al, (2020). Ustilaginoidea virens: insights into an emerging rice pathogen. Annu Rev Phytopathol. https://doi.org/10.1146/annurev-phyto-010820-012908

Sun WB, Wang A, Xu D, Wang WX, Meng JJ, Dai JG et al, (2017). New ustilaginoidins from rice false smut balls caused by Villosiclava virens and their phytotoxic and cytotoxic activities. J Agric Food Chem 65, (25). https://doi.org/10.1021/acs.jafc.7b01791

Tang J, Bai J, Chen X, Zheng L, Liu H, Huang J, (2019). Two protein kinases UvPmk1 and $\mathrm{UvCdc} 2$ with significant functions in conidiation, stress response and pathogenicity of rice false smut fungus Ustilaginoidea virens. Curr Genet. https://doi.org/10.1007/s00294-019-01029-y

Teng X, Yau E, Sing C, Hardwick JM, (2018). Whi2 signals low leucine availability to halt yeast growth and cell death. FEMS Yeast Res 18, (8). https://doi.org/10.1093/femsyr/foy095

Timpano H, Chan Ho Tong L, Gautier V, Lalucque H, Silar P, (2016). The PaPsrl and $P a W h i 2$ genes are members of the regulatory network that connect stationary phase to mycelium differentiation and reproduction in Podospora anserina. Fungal Genet Biol 94: 1-10. https://doi.org/10.1016/j.fgb.2016.06.006

Wang X, Wang J, Lai D, Wang W, Dai J, Zhou L et al, (2017). Ustiloxin G, a new cyclopeptide mycotoxin from rice false smut balls. Toxins (Basel) 9, (2). https://doi.org/10.3390/toxins9020054

Wang Y, Wang F, Xie S, Liu Y, Qu J, Huang J et al, (2019). Development of rice conidiation media for Ustilaginoidea virens. PLoS One 14, (10): e0217667. https://doi.org/10.1371/journal.pone.0217667

Xie S, Wang Y, Wei W, Li C, Liu Y, Qu J et al, (2019). The Bax inhibitor UvBI-1, a negative regulator of mycelial growth and conidiation, mediates stress response and is critical for pathogenicity of the rice false smut fungus Ustilaginoidea virens. Curr Genet 65, (5): 1185-97. 10.1007/s00294-019-00970-2

Xiong M, Meng S, Qiu JH, Shi HB, Shen XL, Kou YJ, (2020). Putative phosphatase UvPsr1 is required for mycelial growth, conidiation, stress response and pathogenicity in Ustilaginonidea virens. Rice Science 27, (6): 529-36. https://doi.org/10.1016/j.rsci.2020.09.009

Yu J, Yu M, Song T, Cao H, Pan X, Yong M et al, (2019). A homeobox transcription factor UvHox2 regulates chlamydospore formation, conidiogenesis, and pathogenicity in Ustilaginoidea virens. Front Microbiol 10: 1071. https://doi.org/10.3389/fmicb.2019.01071

Yu M, Yu J, Hu J, Huang L, Wang Y, Yin X et al, (2015). Identification of pathogenicityrelated genes in the rice pathogen Ustilaginoidea virens through random 
insertional mutagenesis. Fungal Genet Biol 76: 10-9. https://doi.org/10.1016/j.fgb.2015.01.004

Zheng D, Wang Y, Han Y, Xu JR, Wang C, (2016). UvHOG1 is important for hyphal

606 growth and stress responses in the rice false smut fungus Ustilaginoidea virens.

607 Sci Rep 6: 24824. https://doi.org/10.1038/srep24824

608

Zheng MT, Ding H, Huang L, Wang YH, Yu MN, Zheng R et al, (2017). Low-affinity

609 iron transport protein Uvt3277 is important for pathogenesis in the rice false

610 smut fungus Ustilaginoidea virens. Curr Genet 63, (1): 131-44. https://doi.org/10.1007/s00294-016-0620-4

612

613 
a
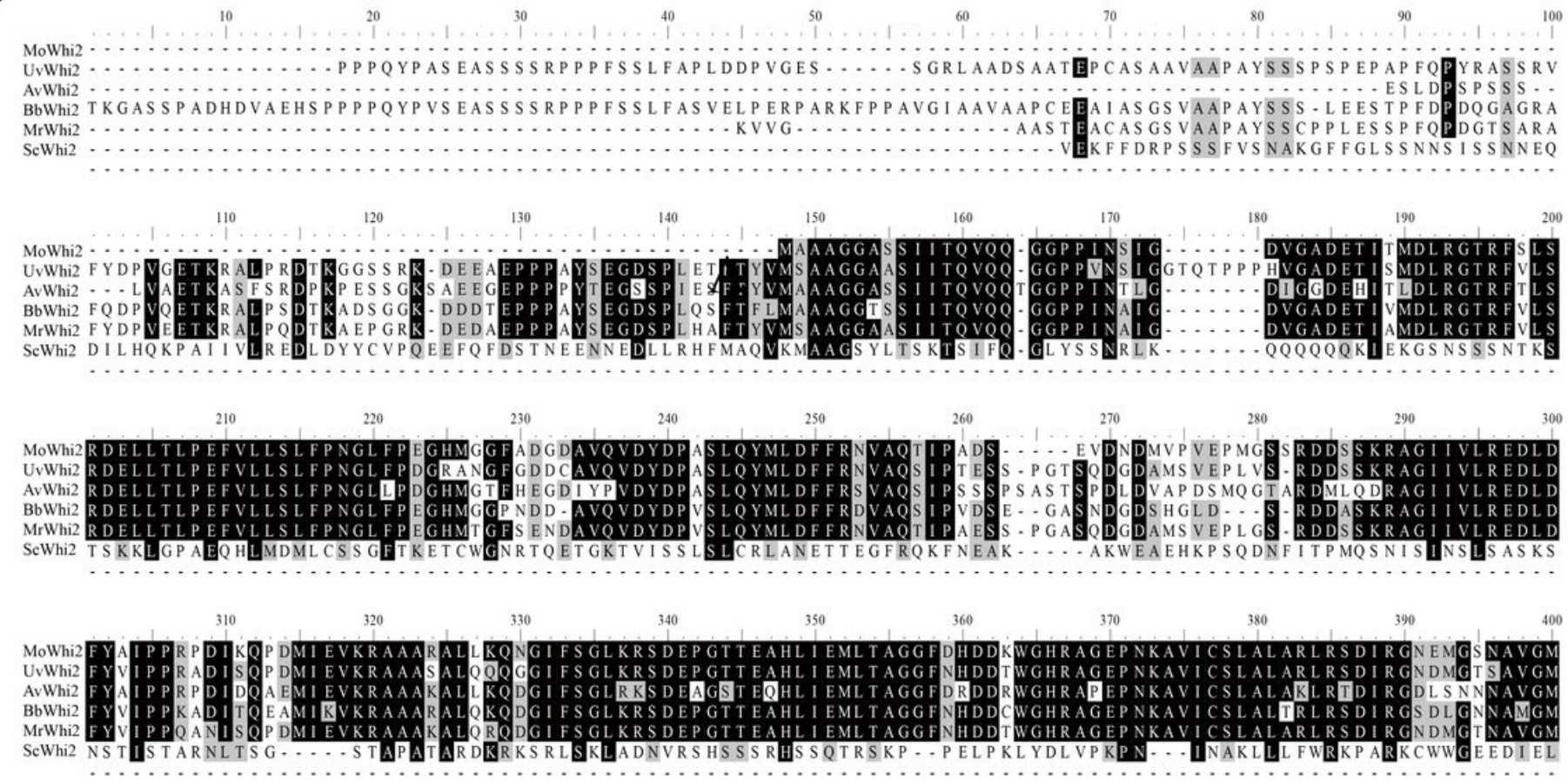

$410-420$

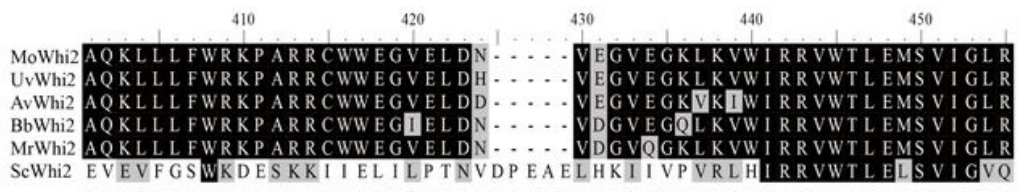

b

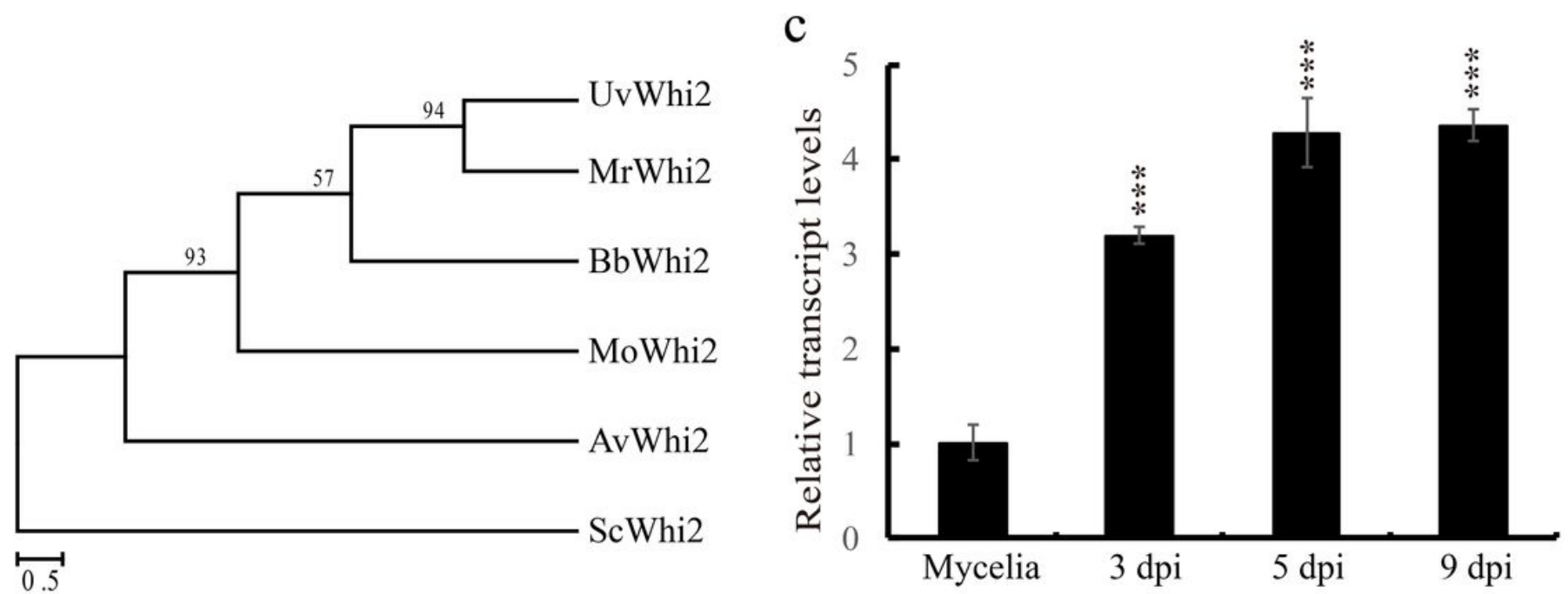

Figure 1

Identification of UvWhi2 in Ustilaginoidea virens. a, The multiple alignment of amino acid sequences of UvWhi2, MrWhi2, BbWhi2, MoWhi2, AvWhi2 and ScWhi2. Amino acids in black and gray represent amino acids identity and fifty percent similarity, respectively. b, Phylogeny analysis of Whi2 orthologs from different fungi was constructed by MEGA 5.0 using the neighbor-joining method. The sequences included 
UvWhi2 (U . virens, KDB15335.1), MrWhi2 (Metarhizium robertsii, XM_007820024.1), BbWhi2 (Beauveria bassiana, XM_008598486.1), MoWhi2 (Magnaporthe oryzae, XP_003714147.1), AvWhi2 (Aspergillus vadensis, XM_025709355.1), and ScWhi2 (Saccharomyces cerevisiae, NP_014686). c, The transcriptional analysis of UvWHI2 in the mycelia and inoculated panicles of WanXian 98 (oryza sativa L. indica) at 3, 5, and $9 \mathrm{dpi}$. The data represents the mean \pm SD from three independent replicates. The data were subject

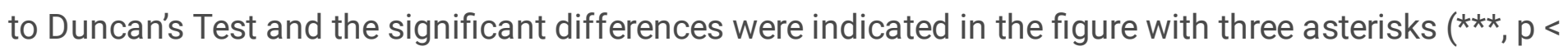
$0.001)$.

a
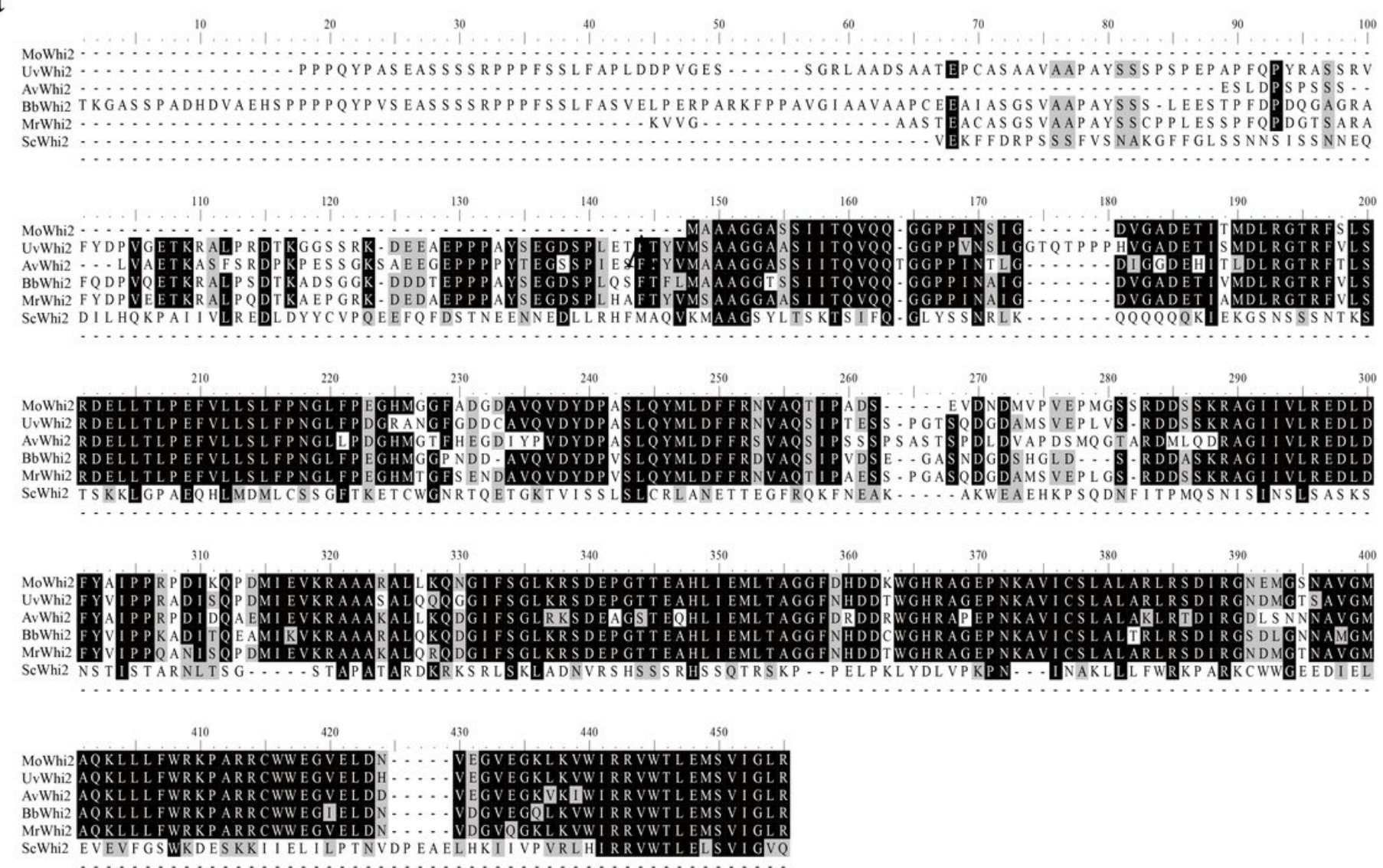

b
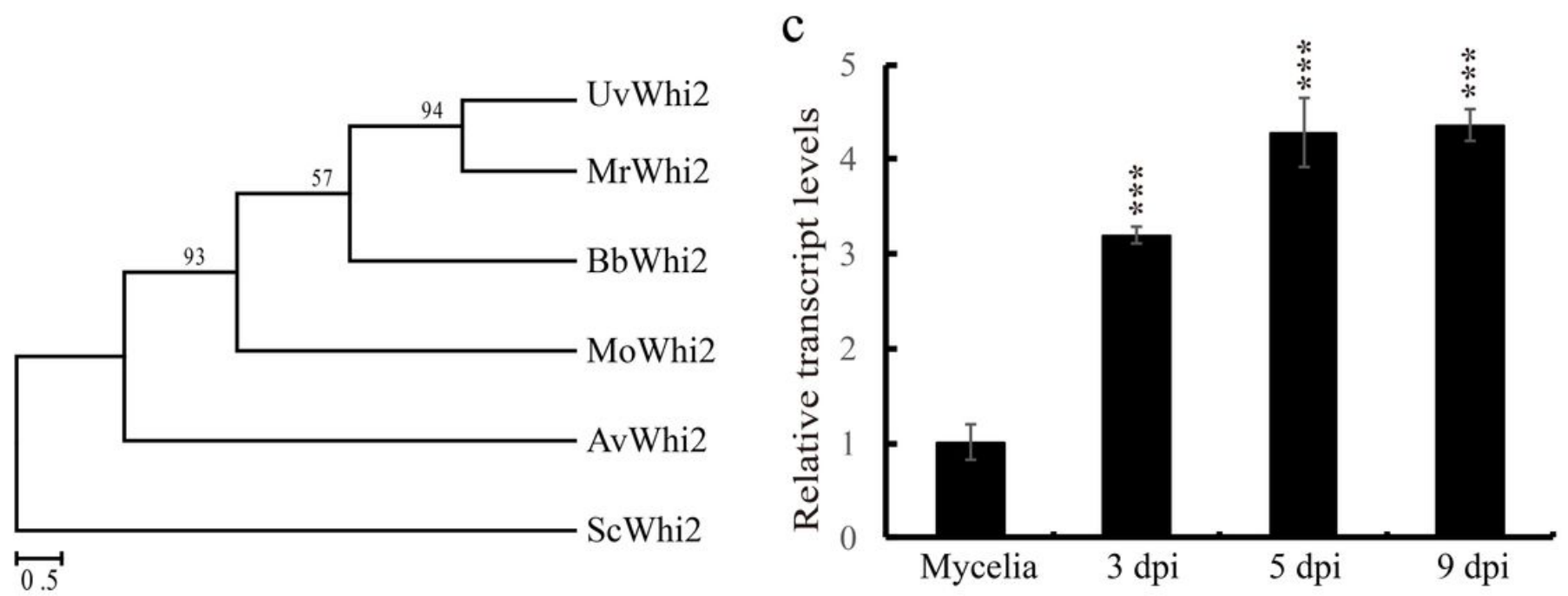

Figure 1 
Identification of UvWhi2 in Ustilaginoidea virens. a, The multiple alignment of amino acid sequences of UvWhi2, MrWhi2, BbWhi2, MoWhi2, AvWhi2 and ScWhi2. Amino acids in black and gray represent amino acids identity and fifty percent similarity, respectively. b, Phylogeny analysis of Whi2 orthologs from different fungi was constructed by MEGA 5.0 using the neighbor-joining method. The sequences included UvWhi2 (U . virens, KDB15335.1), MrWhi2 (Metarhizium robertsii, XM_007820024.1), BbWhi2 (Beauveria bassiana, XM_008598486.1), MoWhi2 (Magnaporthe oryzae, XP_003714147.1), AvWhi2 (Aspergillus vadensis, XM_025709355.1), and ScWhi2 (Saccharomyces cerevisiae, NP_014686). c, The transcriptional analysis of UvWHI2 in the mycelia and inoculated panicles of WanXian 98 (oryza sativa L. indica) at 3, 5, and $9 \mathrm{dpi}$. The data represents the mean \pm SD from three independent replicates. The data were subject to Duncan's Test and the significant differences were indicated in the figure with three asterisks $(\star \star \star, \mathrm{p}<$ $0.001)$. 

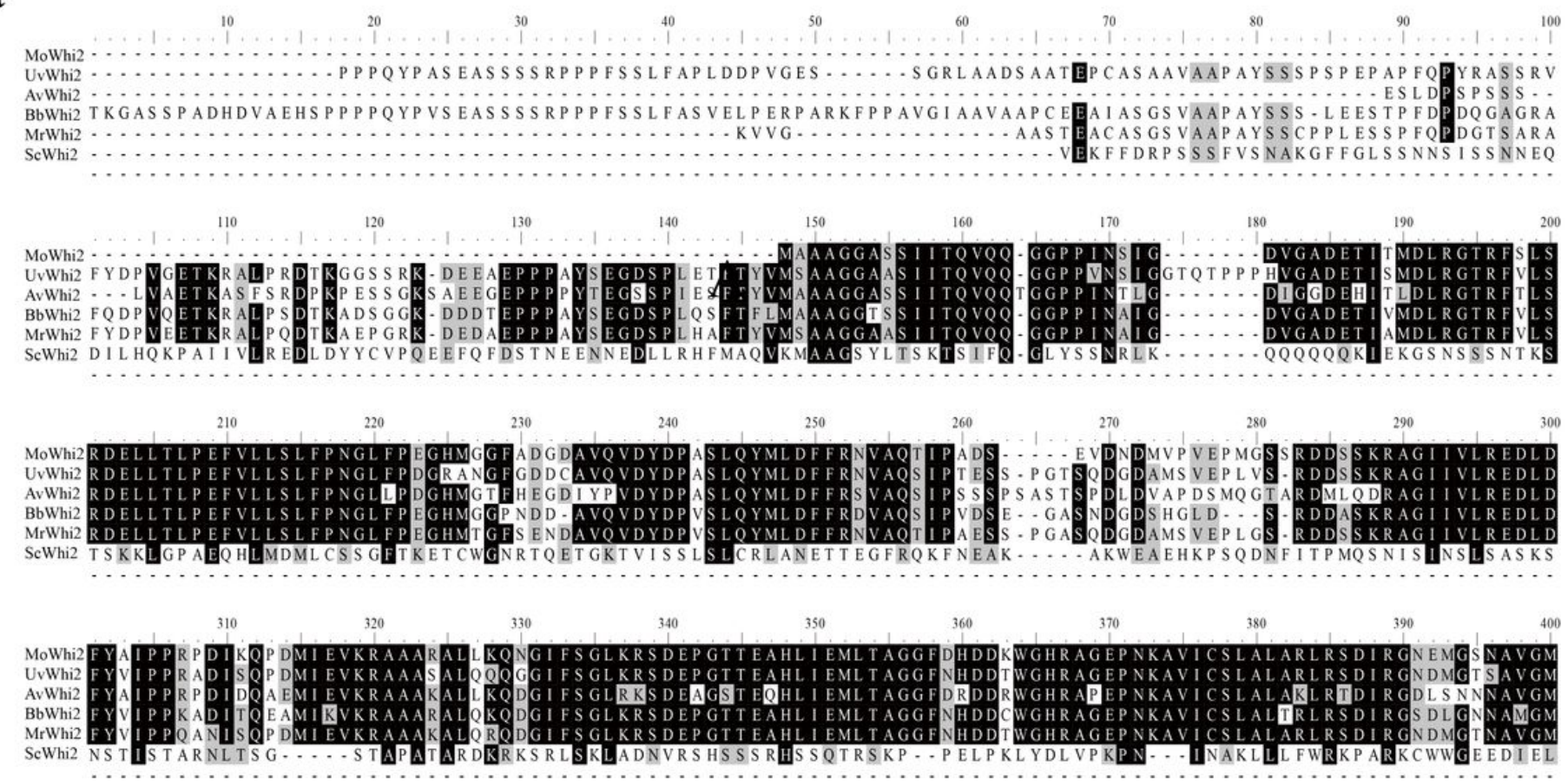

410

420

430

440

450

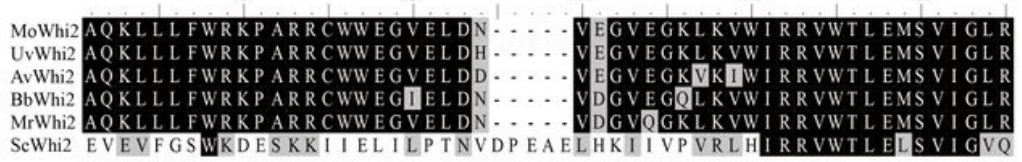

b
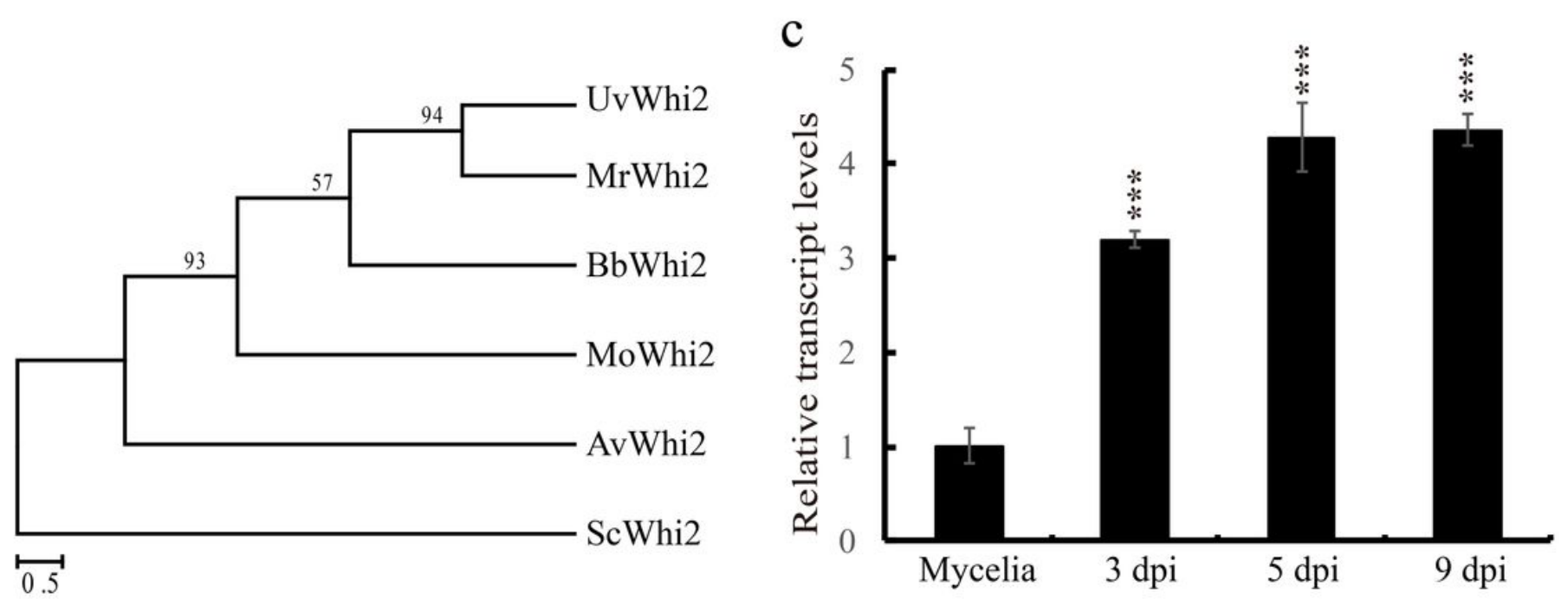

Figure 1

Identification of UvWhi2 in Ustilaginoidea virens. a, The multiple alignment of amino acid sequences of UvWhi2, MrWhi2, BbWhi2, MoWhi2, AvWhi2 and ScWhi2. Amino acids in black and gray represent amino acids identity and fifty percent similarity, respectively. b. Phylogeny analysis of Whi2 orthologs from different fungi was constructed by MEGA 5.0 using the neighbor-joining method. The sequences included UvWhi2 (U . virens, KDB15335.1), MrWhi2 (Metarhizium robertsii, XM_007820024.1), BbWhi2 (Beauveria bassiana, XM_008598486.1), MoWhi2 (Magnaporthe oryzae, XP_003714147.1), AvWhi2 (Aspergillus 
vadensis, XM_025709355.1), and ScWhi2 (Saccharomyces cerevisiae, NP_014686). c, The transcriptional analysis of UVWHI2 in the mycelia and inoculated panicles of WanXian 98 (oryza sativa L. indica) at 3, 5, and $9 \mathrm{dpi}$. The data represents the mean \pm SD from three independent replicates. The data were subject to Duncan's Test and the significant differences were indicated in the figure with three asterisks ( $\star \star \star, p<$ $0.001)$.

$\mathrm{a}$

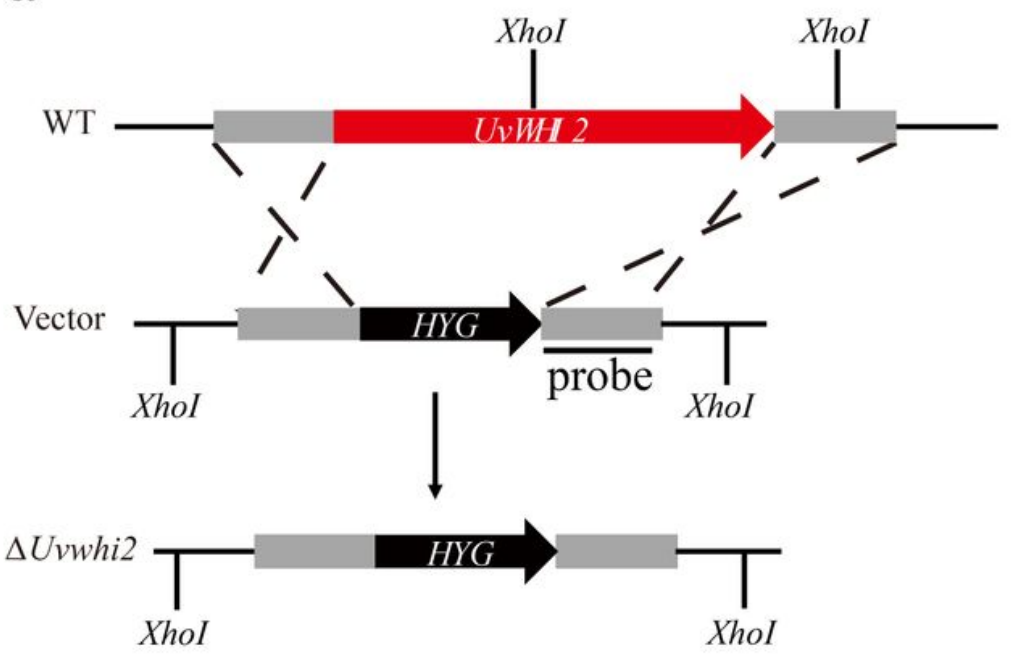

b

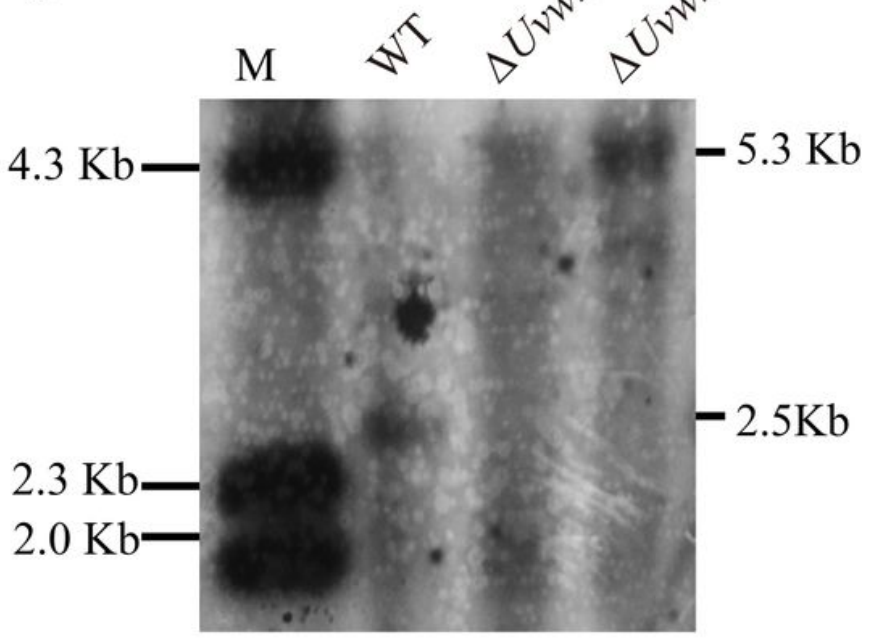

$\mathrm{d}$

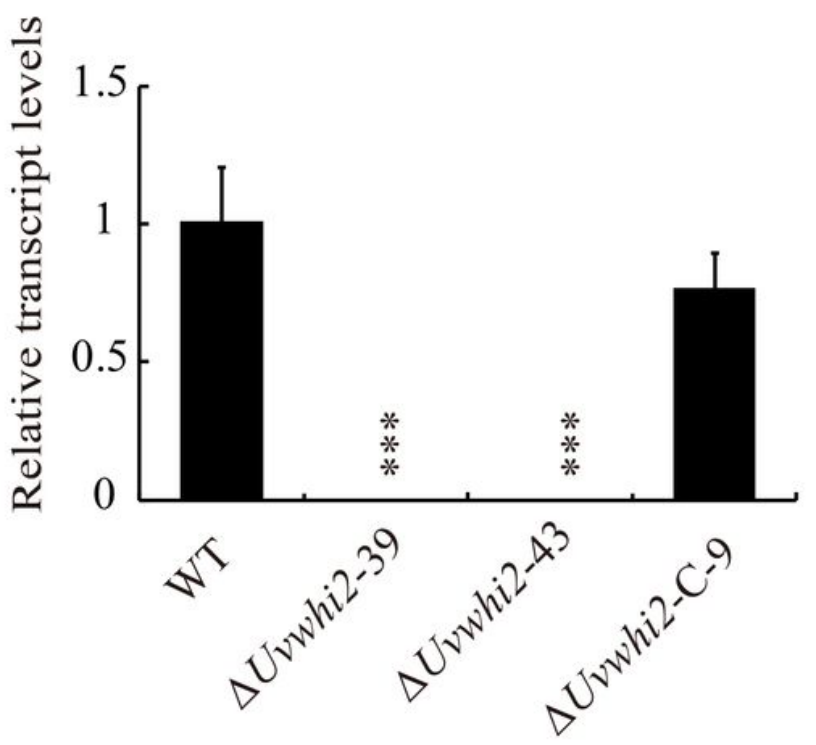

Figure 2

Targeted gene deletion of UvWHI2 and complementation assay in U. virens. a, Construction strategy for the UvWHI2 gene deletion mutant in U. virens. The coding region of UvWHI2 was replaced with the hygromycin phosphotransferase gene cassette (HYG) by homologous recombination. b, The Southern blot assay was used to validate the loss of UvWHI2 in the $\triangle$ Uvwhi2 deletion mutant. The Xho I enzyme was used to digested genomic DNA of the WT and $\triangle$ Uvwhi2 strains. The digested genomic were processed for the Southern blotting with the $1 \mathrm{~Kb}$ downstream of UvWHI2 as probe. c, qRT-PCR analysis 
of the expression level of UvWHI2 in the WT, $\Delta$ Uvwhi2, and $\Delta$ Uvwhi2-C strains. The data represents the mean \pm SD from three independent replicates. The asterisks (***) indicate a $P$ value $<0.001$.

a

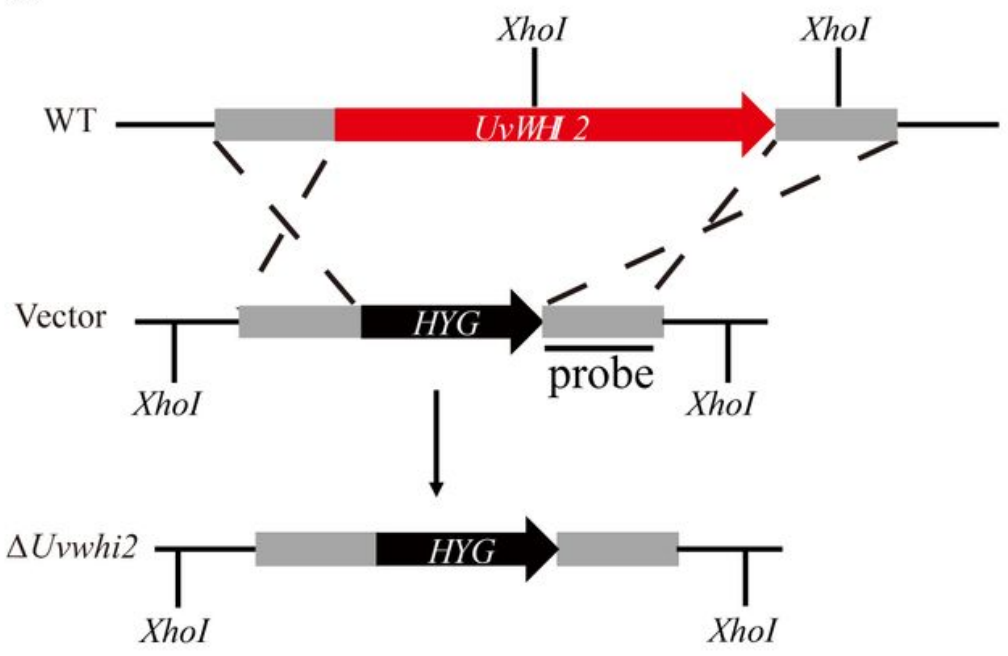

$\mathrm{b}$

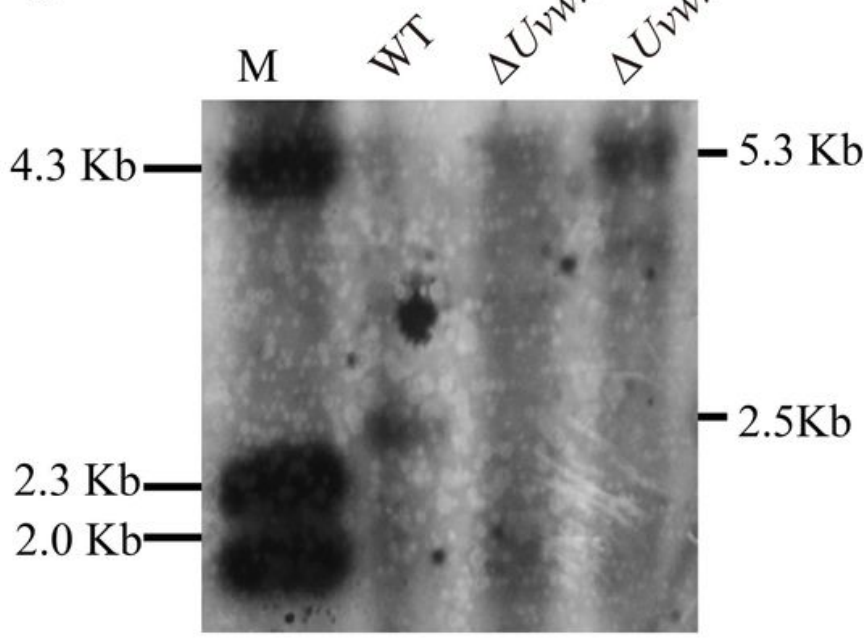

d

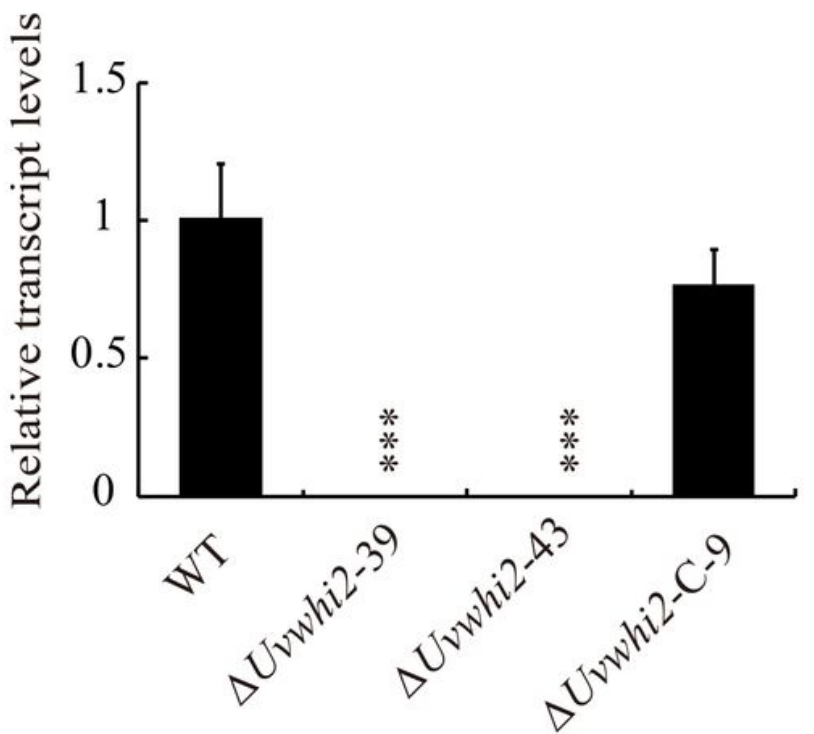

Figure 2

Targeted gene deletion of UvWHI2 and complementation assay in U. virens. a, Construction strategy for the UvWHI2 gene deletion mutant in U. virens. The coding region of UvWHI2 was replaced with the hygromycin phosphotransferase gene cassette (HYG) by homologous recombination. b, The Southern blot assay was used to validate the loss of UvWHI2 in the $\Delta$ Uvwhi2 deletion mutant. The Xho I enzyme was used to digested genomic DNA of the WT and $\Delta$ Uvwhi2 strains. The digested genomic were processed for the Southern blotting with the $1 \mathrm{~Kb}$ downstream of UvWHI2 as probe. C, qRT-PCR analysis of the expression level of UvWHI2 in the WT, $\Delta$ Uvwhi2, and $\Delta$ Uvwhi2-C strains. The data represents the mean \pm SD from three independent replicates. The asterisks $(* \star *)$ indicate a $P$ value $<0.001$. 
a

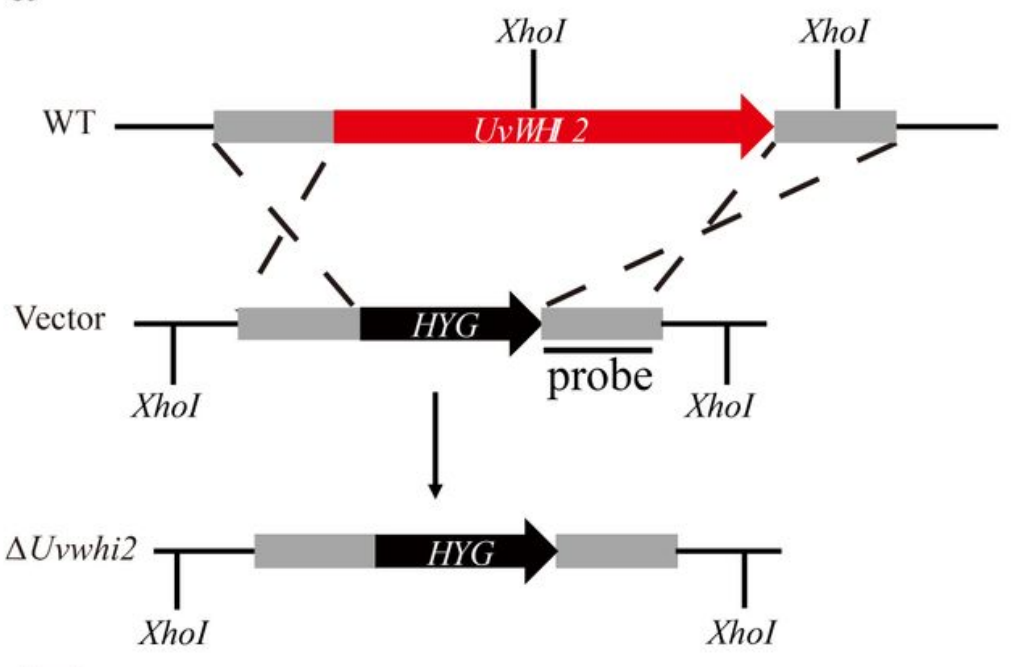

b

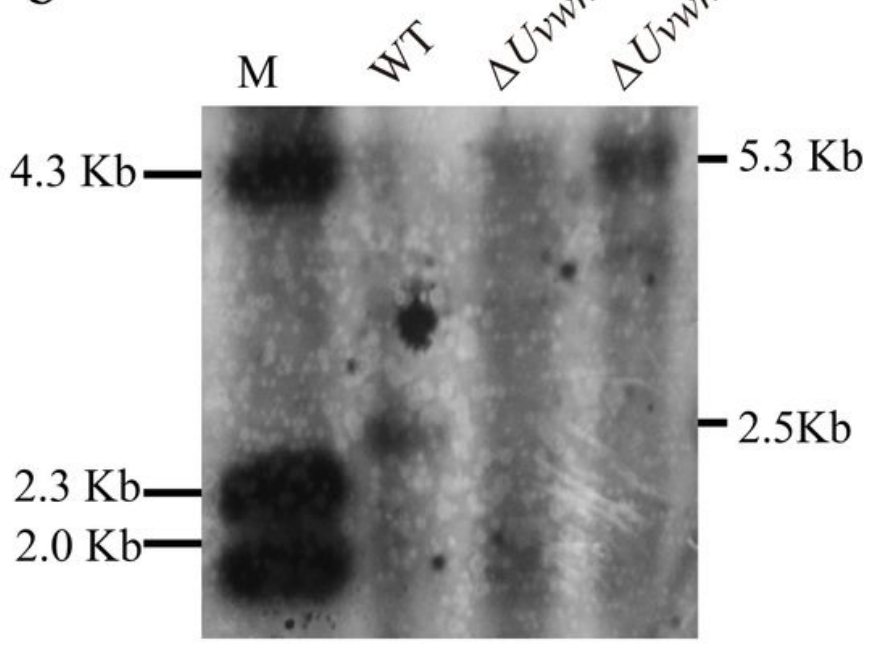

$\mathrm{d}$
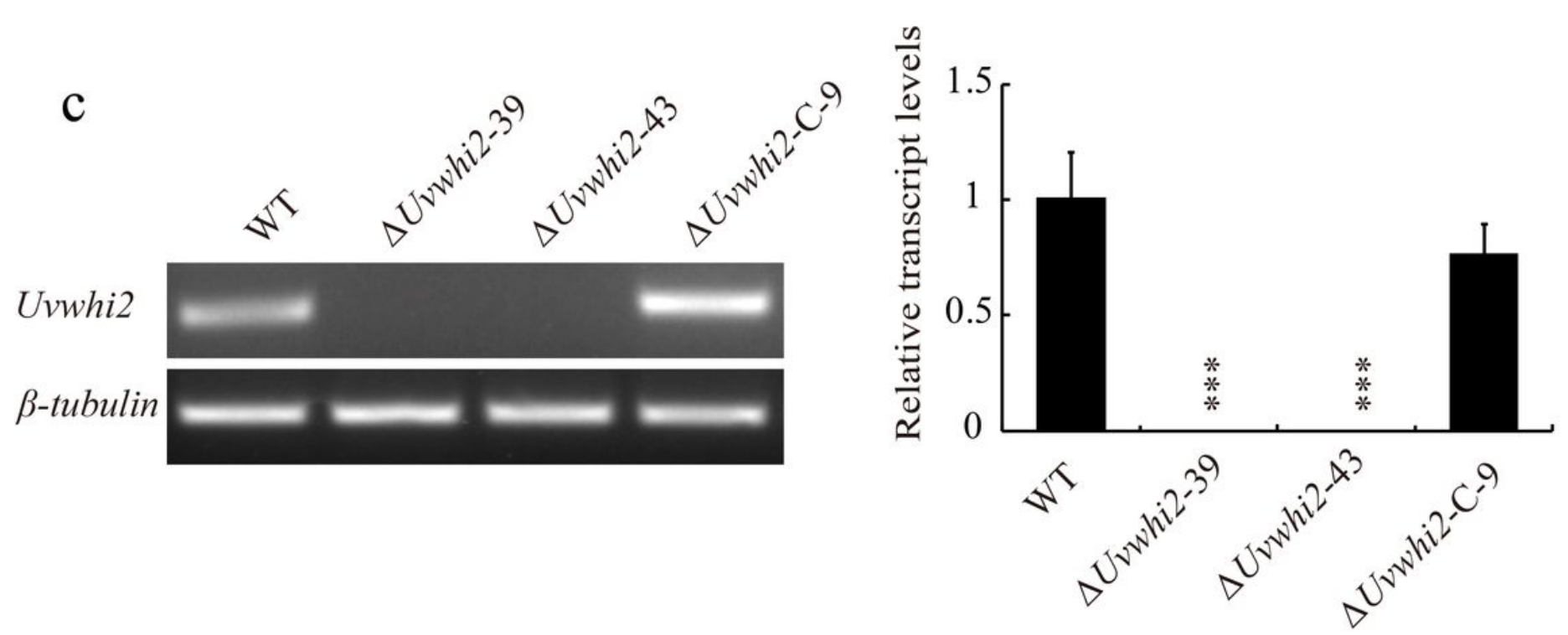

\section{Figure 2}

Targeted gene deletion of UvWHI2 and complementation assay in U. virens. a, Construction strategy for the UvWHI2 gene deletion mutant in U. virens. The coding region of UvWHI2 was replaced with the hygromycin phosphotransferase gene cassette (HYG) by homologous recombination. b, The Southern blot assay was used to validate the loss of UvWHI2 in the $\Delta$ Uvwhi2 deletion mutant. The Xho I enzyme was used to digested genomic DNA of the WT and $\Delta$ Uvwhi2 strains. The digested genomic were processed for the Southern blotting with the $1 \mathrm{~Kb}$ downstream of UvWHI2 as probe. C, qRT-PCR analysis of the expression level of UvWHI2 in the WT, $\Delta$ Uvwhi2, and $\Delta$ Uvwhi2-C strains. The data represents the mean \pm SD from three independent replicates. The asterisks $(* \star \star)$ indicate a P value $<0.001$. 
a
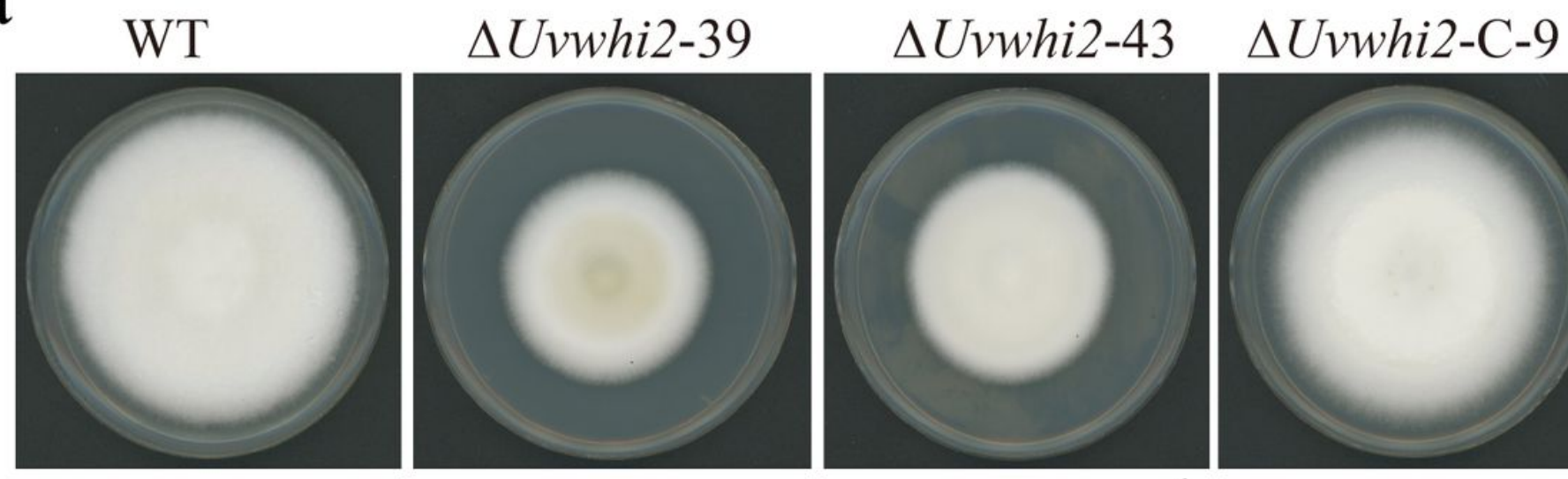

b

$\mathrm{C}$
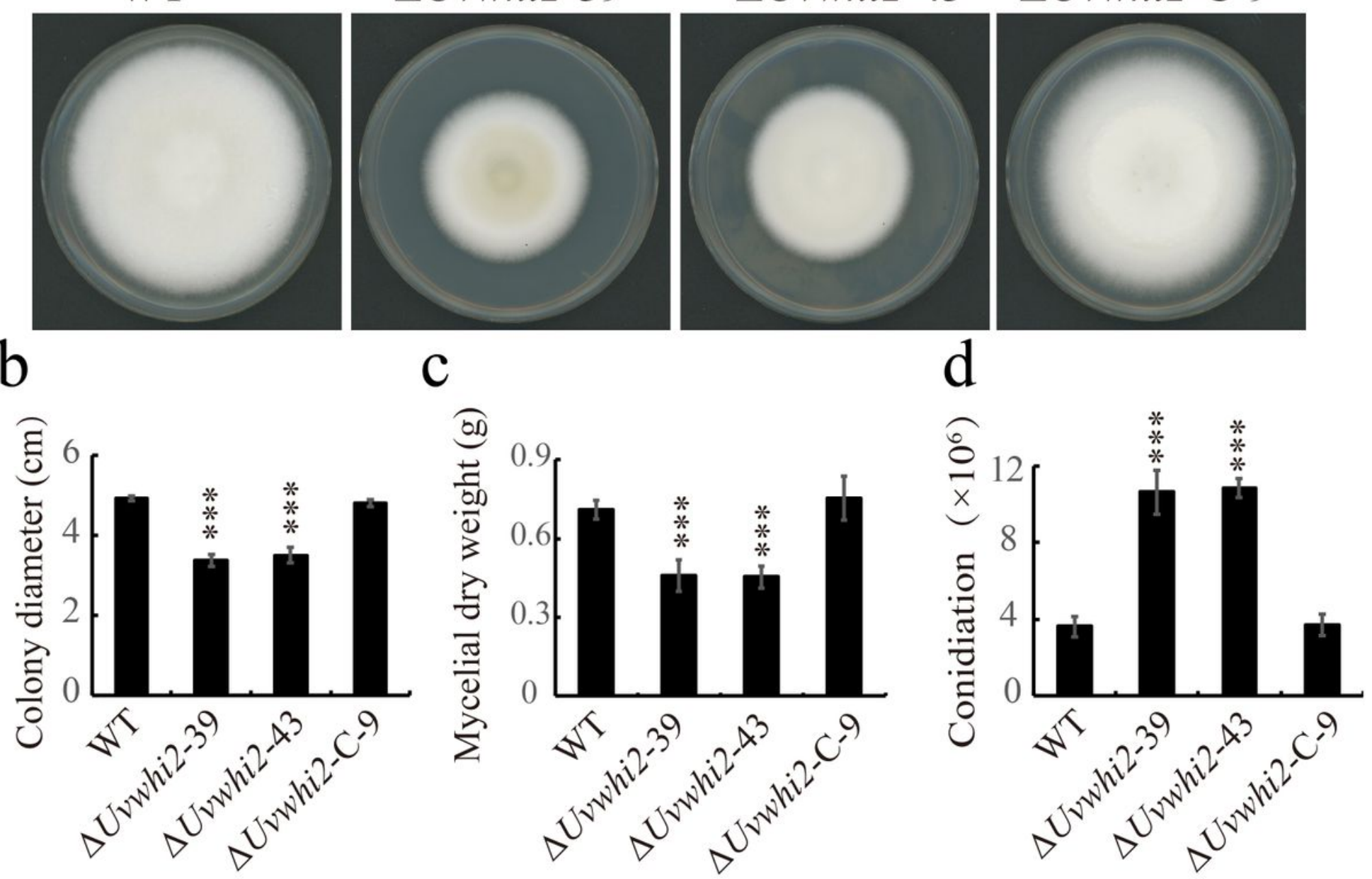

$\mathrm{e}$

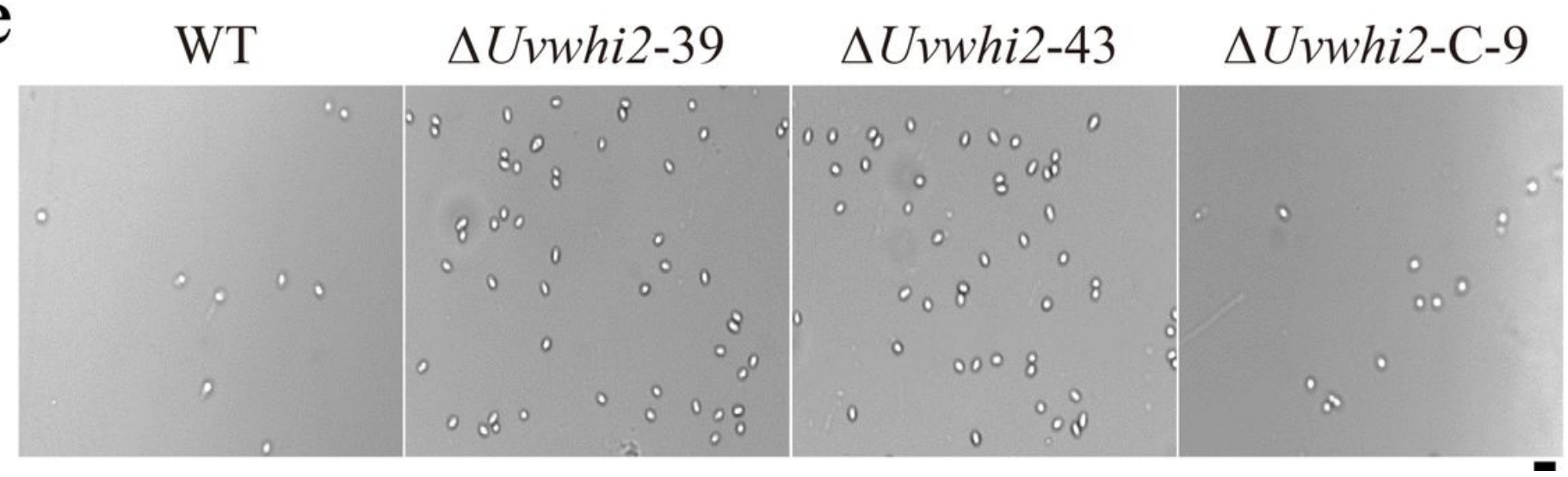

Figure 3

Deletion of UvWHI2 results in decreased vegetative growth and increased conidiation in U. virens. a, Mycelia growth of the WT, $\triangle \mathrm{Uvwhi2}$, and $\triangle \mathrm{Uvwwhi2}-\mathrm{C}$ strains on the PSA (potato sucrose agar) medium in dark at $28 \rrbracket$ for $15 \mathrm{~d}$. b, Colony morphology of indicated strains. c, Dry weight of mycelia was measured in the PS medium after $7 d$ culture. $d$ and e, Knockout of UvWHI2 enhanced conidiation under nutrient-rich condition. Data represents the mean \pm SD from three independent replicates. The data were subject to 
Duncan's Test and the significant differences were indicated in the figure with three asterisks ( $\star \star \star, p<$ 0.001). Scale bar $=5 \mu \mathrm{m}$.
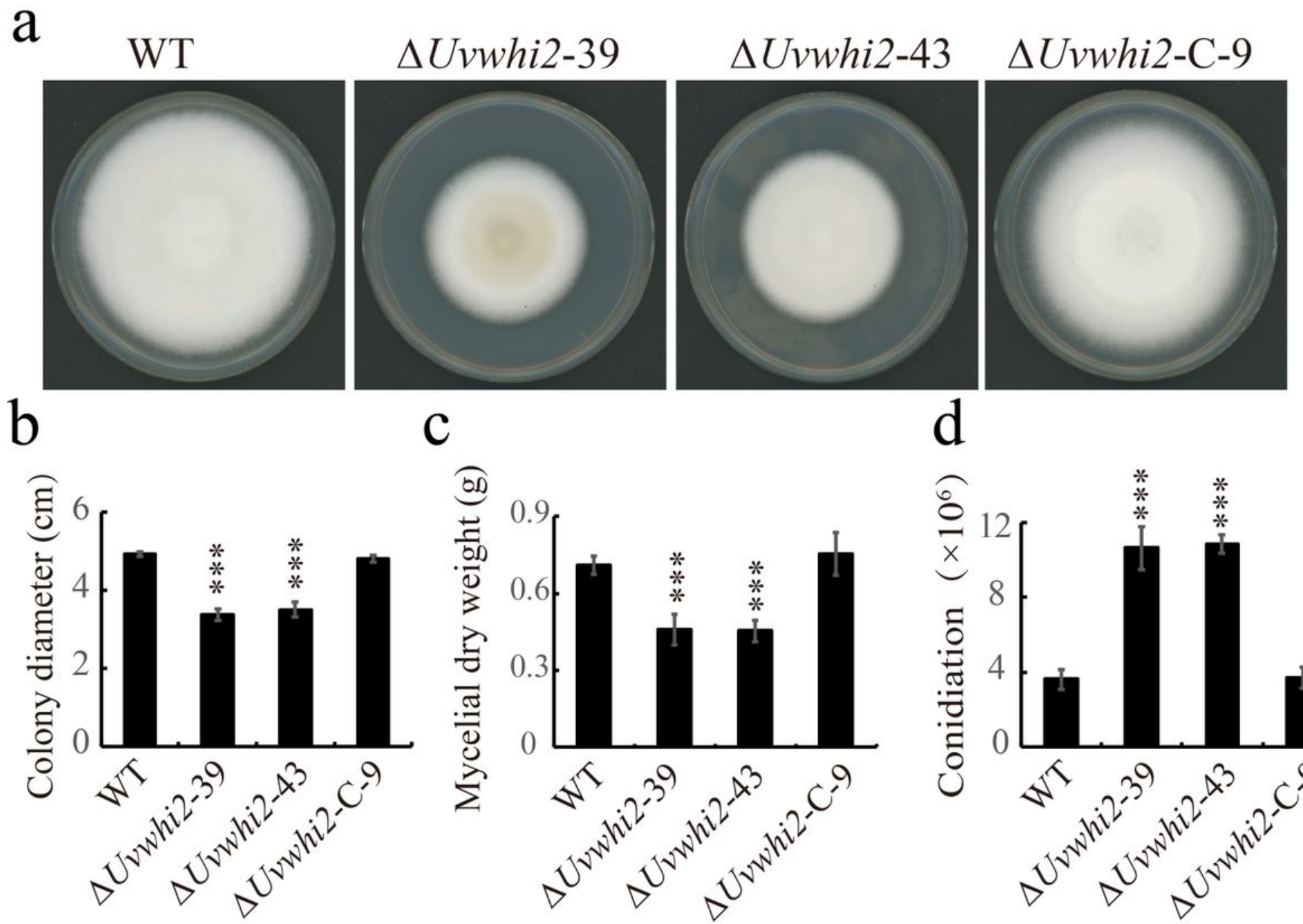

$\mathrm{C}$

e
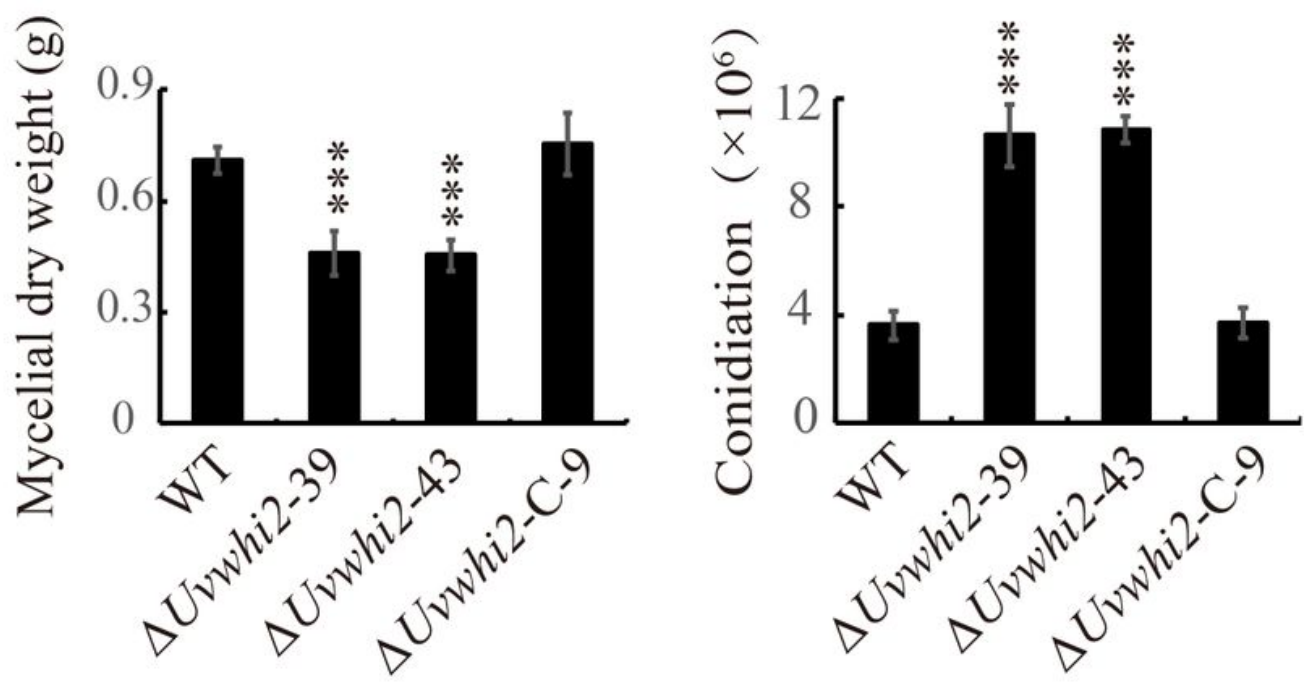
WT $\Delta U v w h i 2-39$

$\Delta U v w h i 2-43$ $\Delta U v w h i 2-\mathrm{C}-9$

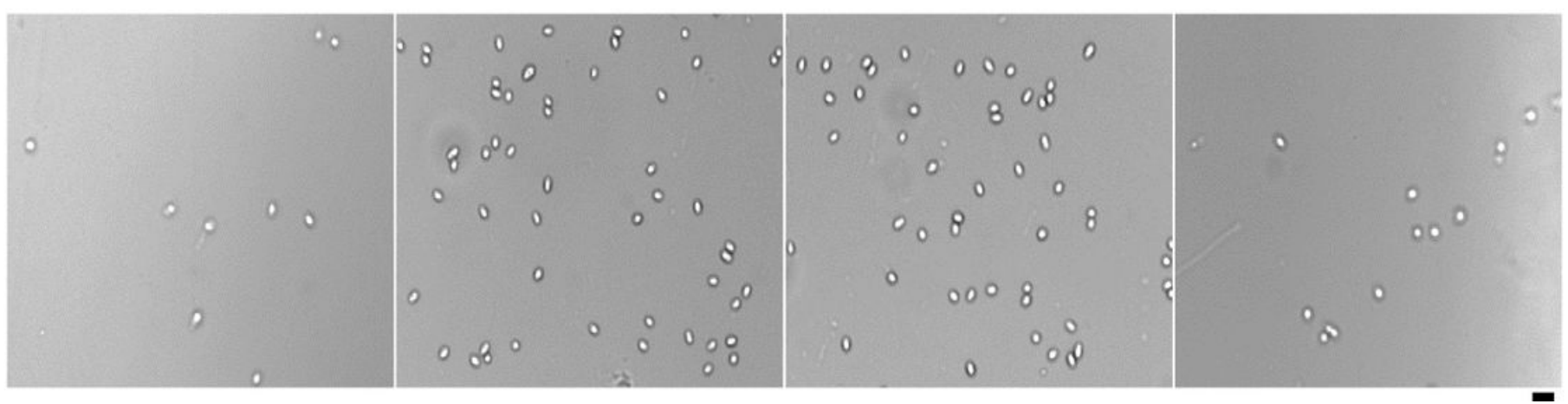

Figure 3

Deletion of UvWHI2 results in decreased vegetative growth and increased conidiation in U. virens. a, Mycelia growth of the WT, $\triangle$ Uvwhi2, and $\triangle$ Uvwhi2-C strains on the PSA (potato sucrose agar) medium in dark at $28 \otimes$ for $15 \mathrm{~d}$. b, Colony morphology of indicated strains. c, Dry weight of mycelia was measured in the PS medium after $7 \mathrm{~d}$ culture. $\mathrm{d}$ and e, Knockout of UvWHI2 enhanced conidiation under nutrient-rich 
condition. Data represents the mean \pm SD from three independent replicates. The data were subject to Duncan's Test and the significant differences were indicated in the figure with three asterisks ( $\star \star \star, p<$ 0.001). Scale bar $=5 \mu \mathrm{m}$.

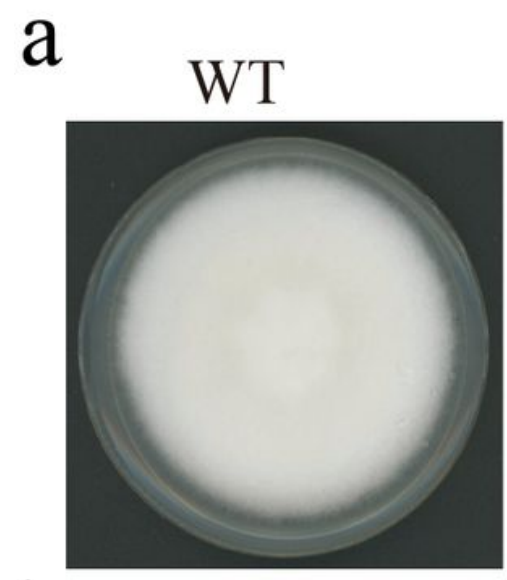

b

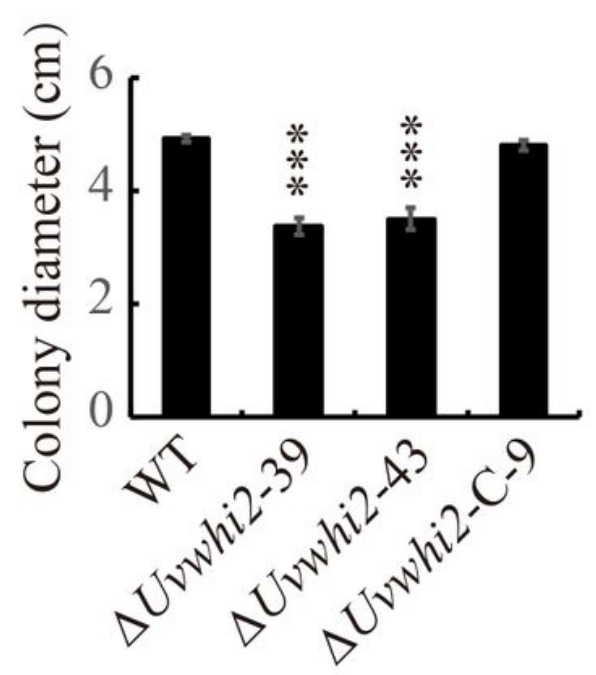

$\Delta U v w h i 2-39$

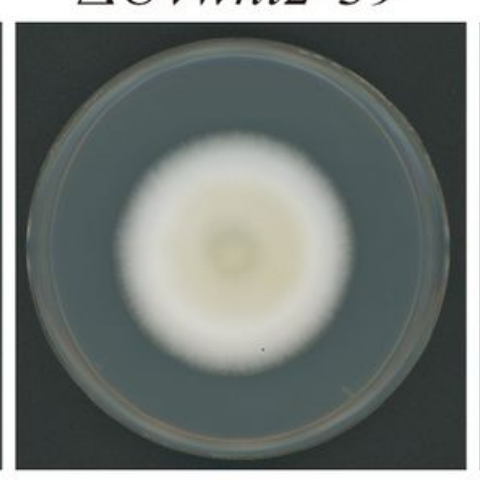

C
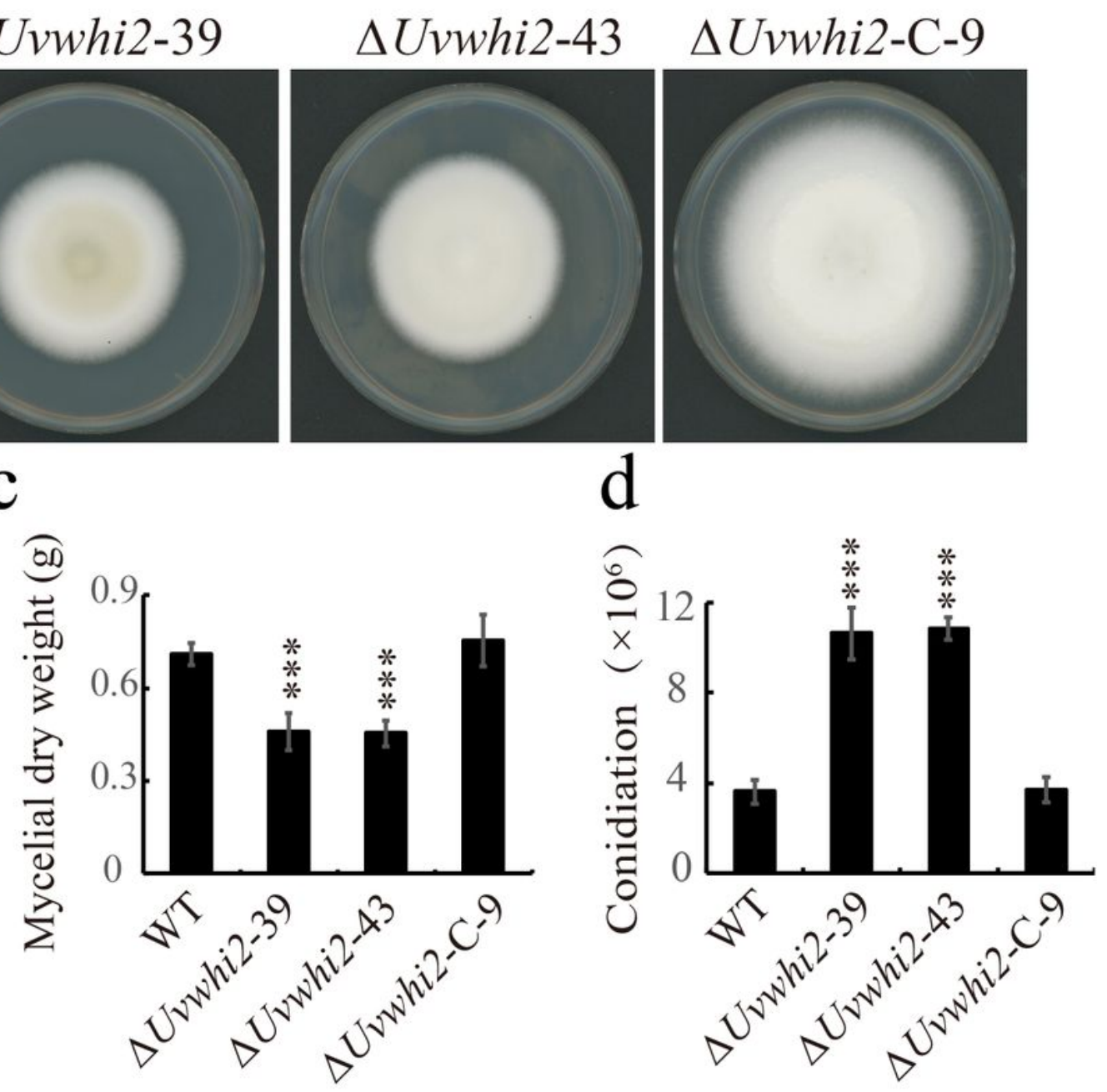

e $\Delta U v w h i 2-39$ $\Delta U v w h i 2-43$ $\Delta U v w h i 2-\mathrm{C}-9$

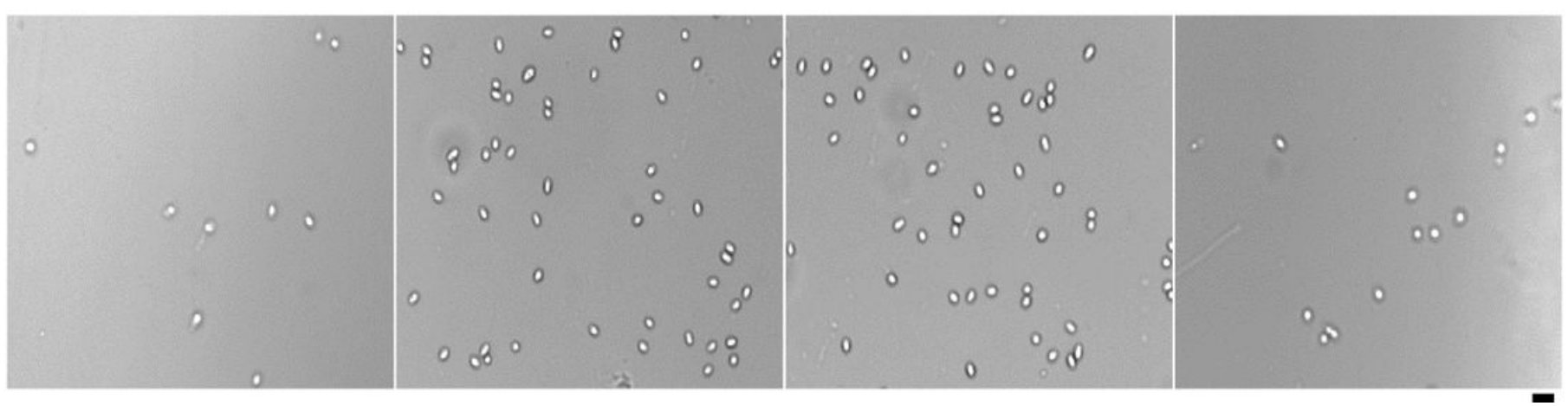

Figure 3

Deletion of UvWHI2 results in decreased vegetative growth and increased conidiation in U. virens. a, Mycelia growth of the WT, $\Delta U v w$ whi2, and $\triangle U v w$ whi2-C strains on the PSA (potato sucrose agar) medium in dark at $28 \rrbracket$ for $15 \mathrm{~d}$. b, Colony morphology of indicated strains. c, Dry weight of mycelia was measured in 
the PS medium after $7 \mathrm{~d}$ culture. $\mathrm{d}$ and e, Knockout of UvWHI2 enhanced conidiation under nutrient-rich condition. Data represents the mean \pm SD from three independent replicates. The data were subject to Duncan's Test and the significant differences were indicated in the figure with three asterisks ( $\star \star \star, p<$ 0.001). Scale bar $=5 \mu \mathrm{m}$.

a

WT $\quad \Delta U v w h i 2-39 \Delta U v w h i 2-43 \quad \Delta U v w h i 2-\mathrm{C}-9$

b
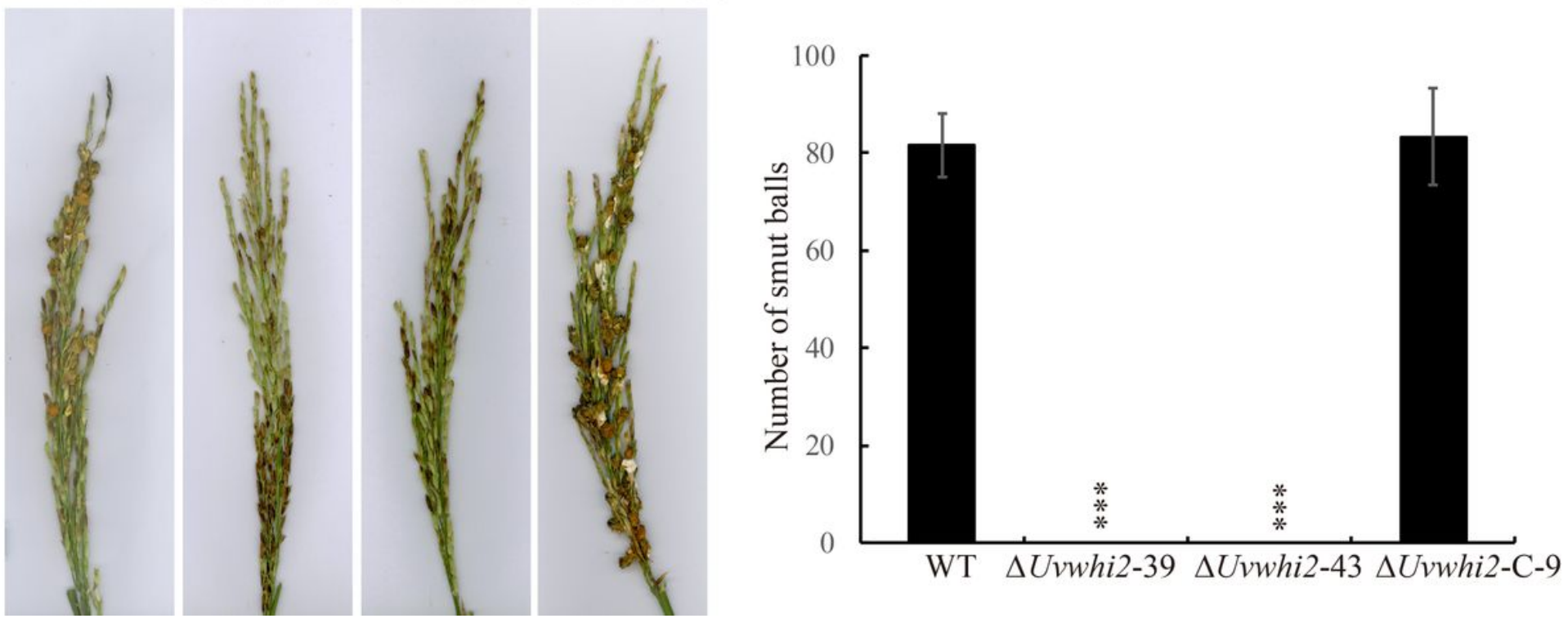

Figure 4

UvWhi2 is required for pathogenesis in U. virens. a, Disease symptoms of indicated strain on the rice panicles of WanXian 98 at $21 \mathrm{dpi}$. b. Statistical analysis of the average number of false smut balls on the inoculated spikelets. Each experiment was performed with three independent biological experiments and more than thirty panicles were inoculated each time. Data were showed as Mean $\pm S D(n=3)$. ***, $p<$ 0.001 .

a

WT $\quad \Delta U v w h i 2-39 \Delta U v w h i 2-43 \Delta U v w h i 2-\mathrm{C}-9$

b
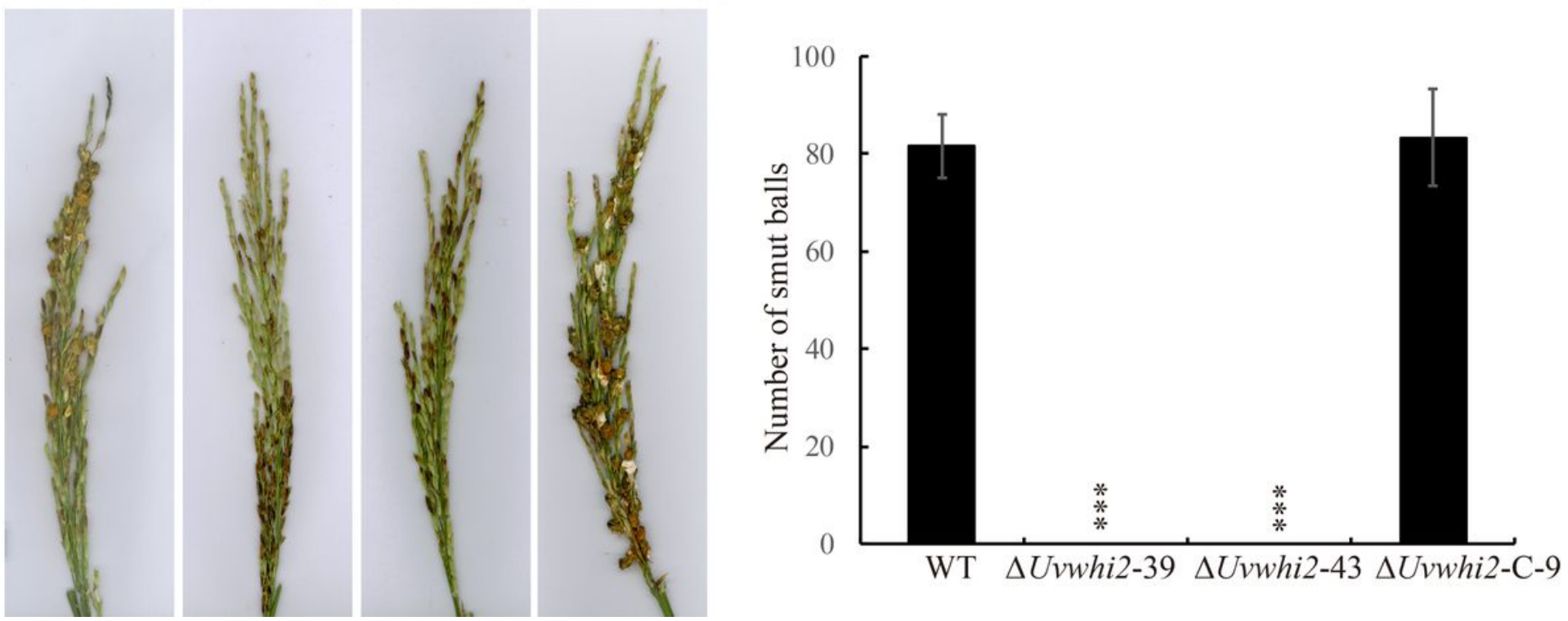

Figure 4 
UvWhi2 is required for pathogenesis in U. virens. a, Disease symptoms of indicated strain on the rice panicles of WanXian 98 at $21 \mathrm{dpi}$. b. Statistical analysis of the average number of false smut balls on the inoculated spikelets. Each experiment was performed with three independent biological experiments and more than thirty panicles were inoculated each time. Data were showed as Mean $\pm \operatorname{SD}(n=3)$. ***, $p<$ 0.001 .

a

WT $\quad \Delta U v w h i 2-39 \quad \Delta U v w h i 2-43 \quad \Delta U v w h i 2-\mathrm{C}-9$

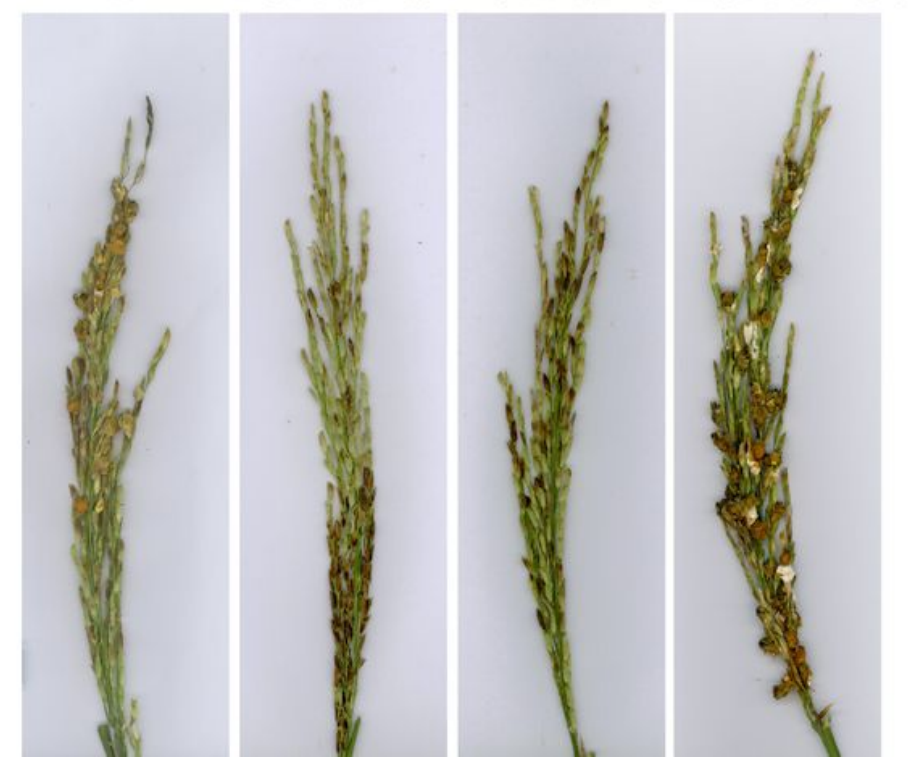

b

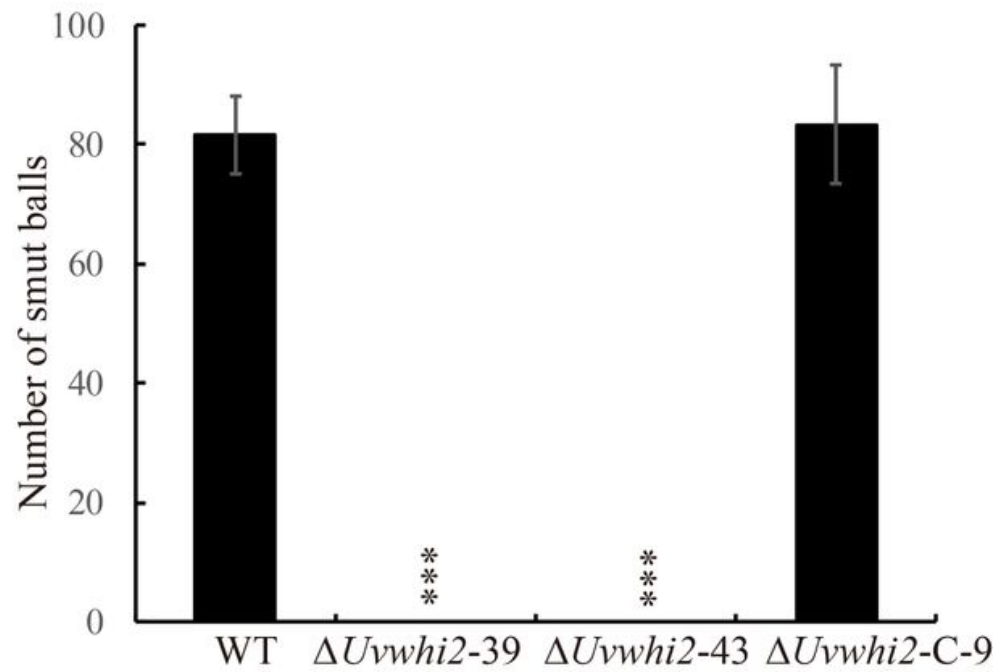

Figure 4

UvWhi2 is required for pathogenesis in U. virens. a, Disease symptoms of indicated strain on the rice panicles of WanXian 98 at $21 \mathrm{dpi}$. b. Statistical analysis of the average number of false smut balls on the inoculated spikelets. Each experiment was performed with three independent biological experiments and more than thirty panicles were inoculated each time. Data were showed as Mean $\pm \operatorname{SD}(n=3)$. ***, $p<$ 0.001 . 
a

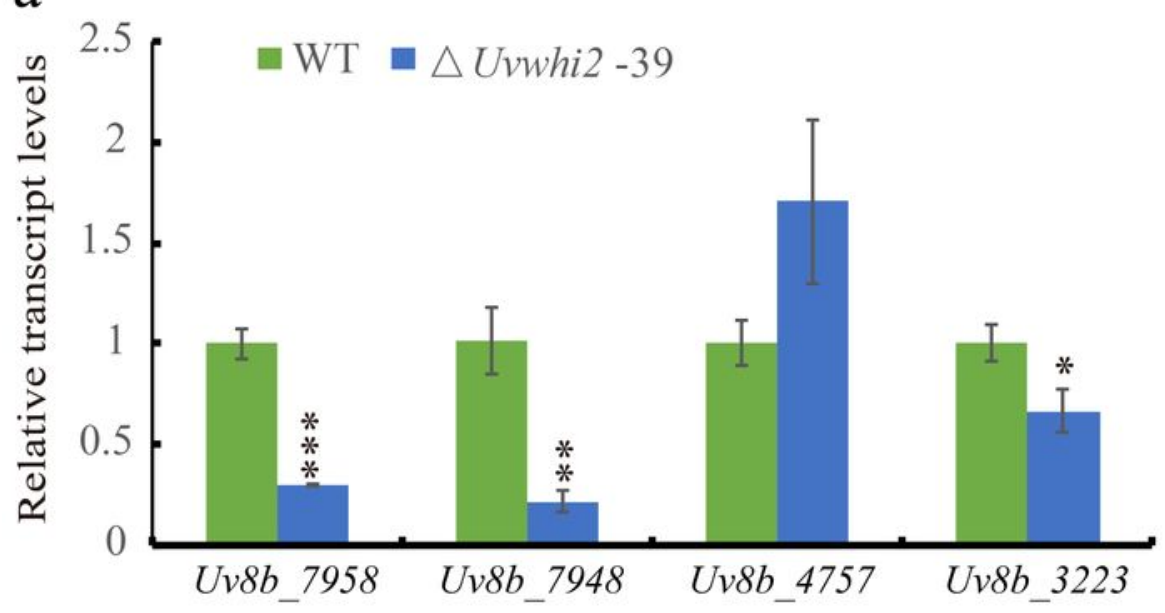

b

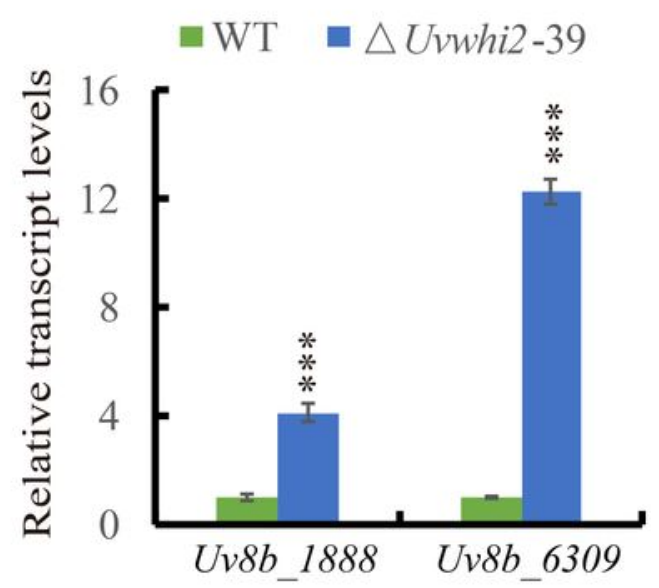

d

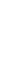

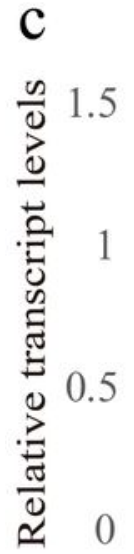

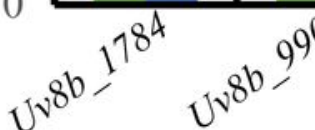

- WT $\triangle U v w h i 2-39$

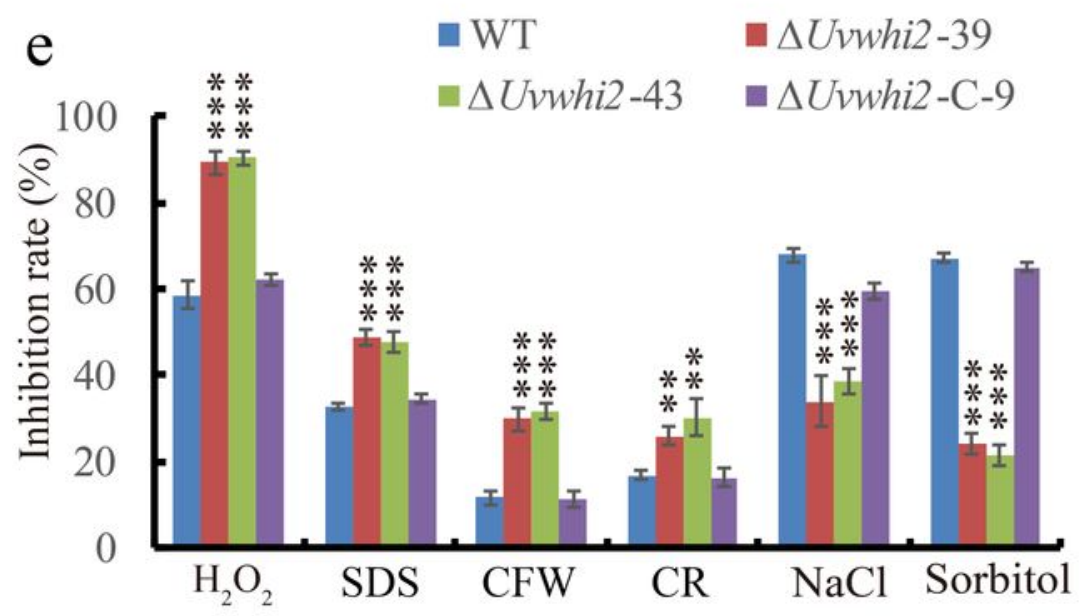
$22^{52}$ * $108 \mathrm{~b}-$

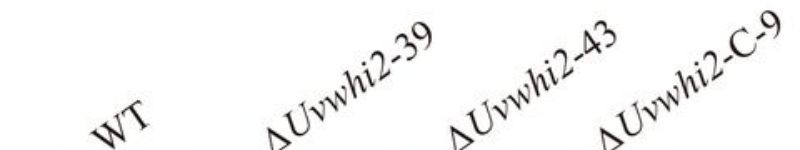


28囚. e, Statistical analysis of inhibition rate of tested strains with different stress agents. The diameters of colonies were measured and calculated. Similar results were obtained by three repeated experiments. The error bars represent the standard deviation and the asterisk represents the significant difference compared to the WT strain under the same conditions ( ${ }^{*}, p<0.01 ; * *, p<0.005 ; * * *, p<0.001$ ).

a
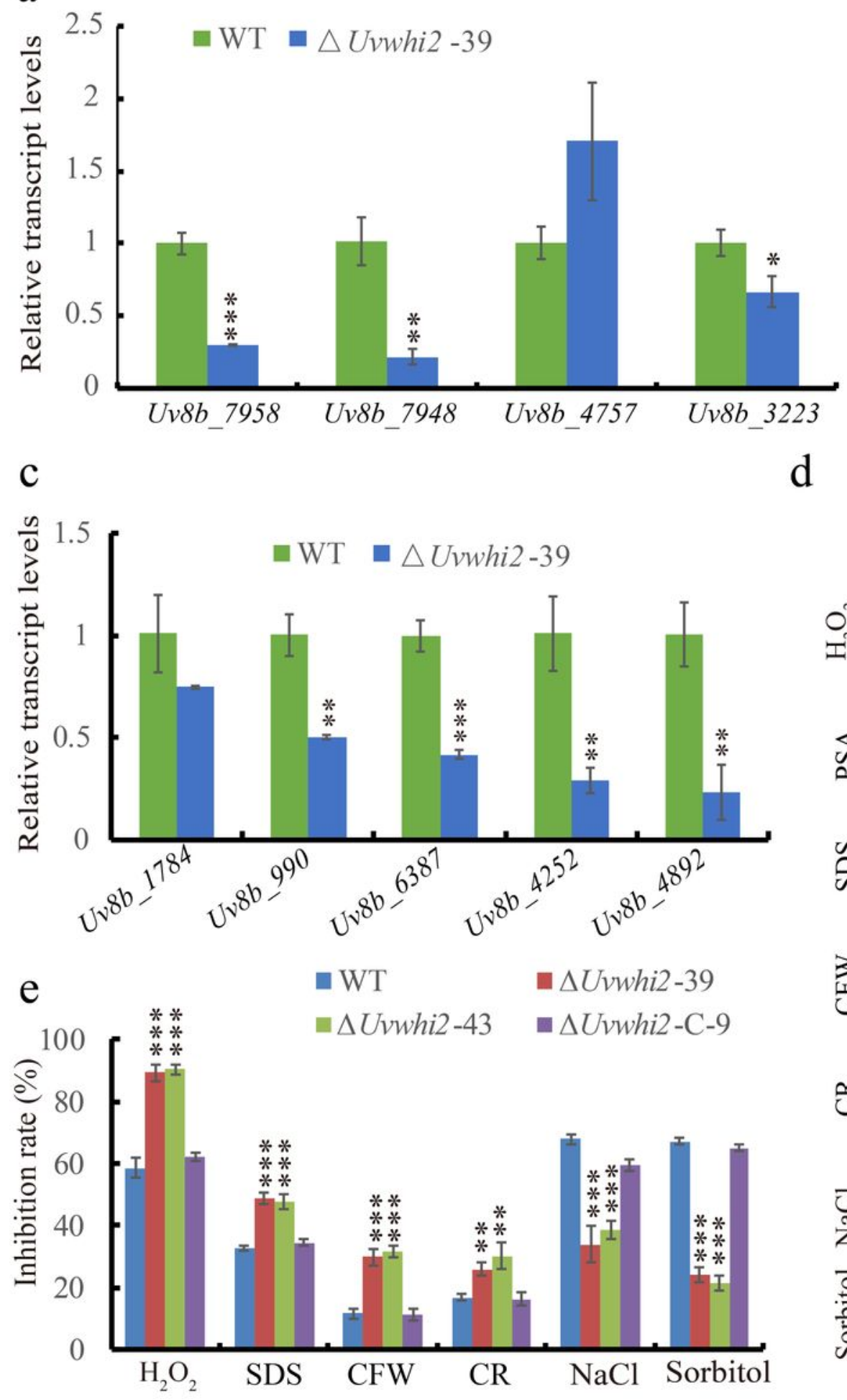

b

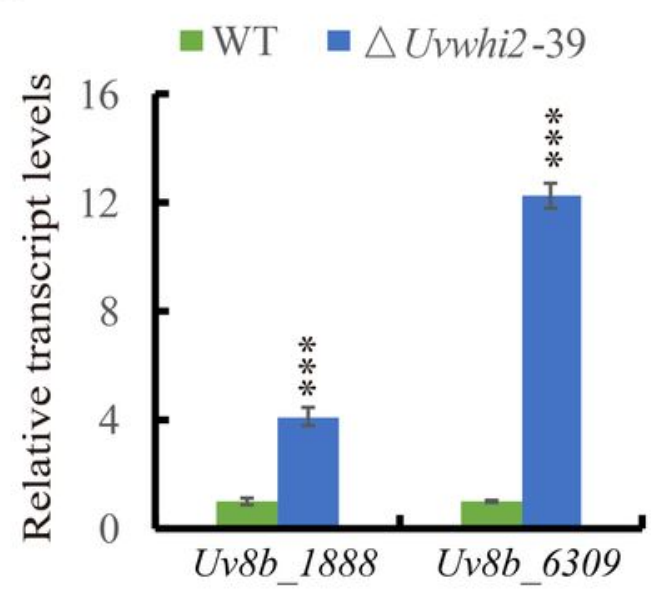

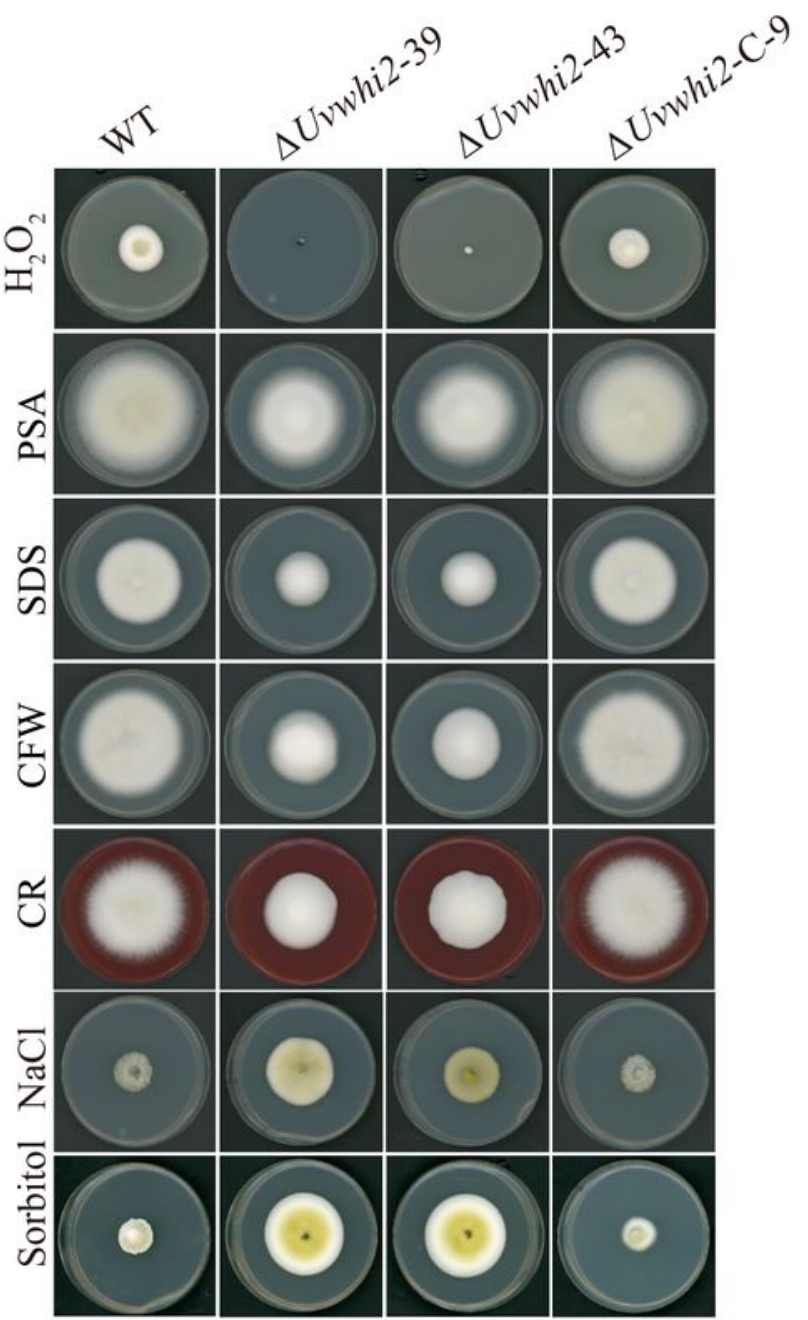

Figure 5 
UvWHI2 contribute to the stress responses to the cell wall, oxidative and osmotic agent in U. virens. $a, b$ and c, qRT-PCR analysis of the expression of the genes related to laccase, peroxidase, chitin deposition and hyperosmotic activities, respectively. $d$, The tested strains grown on the PSA or PSA with $0.03 \% \mathrm{H} 202$ (Oxidative stress agent), $0.03 \%$ SDS (Sodium dodecyl sulfate), $120 \mu \mathrm{g} / \mathrm{mL}$ CFW (Calcofluor white), 120 $\mu \mathrm{g} / \mathrm{mL} \mathrm{CR}$ (Congo red), $0.4 \mathrm{M} \mathrm{NaCl}$, or $0.7 \mathrm{M}$ sorbitol. Typical cultures were photographed after $15 \mathrm{~d}$ at 28凶. e, Statistical analysis of inhibition rate of tested strains with different stress agents. The diameters of colonies were measured and calculated. Similar results were obtained by three repeated experiments. The error bars represent the standard deviation and the asterisk represents the significant difference compared to the WT strain under the same conditions ( $\left.{ }^{*}, \mathrm{p}<0.01 ; * \star, p<0.005 ; \star \star \star, p<0.001\right)$. 
a

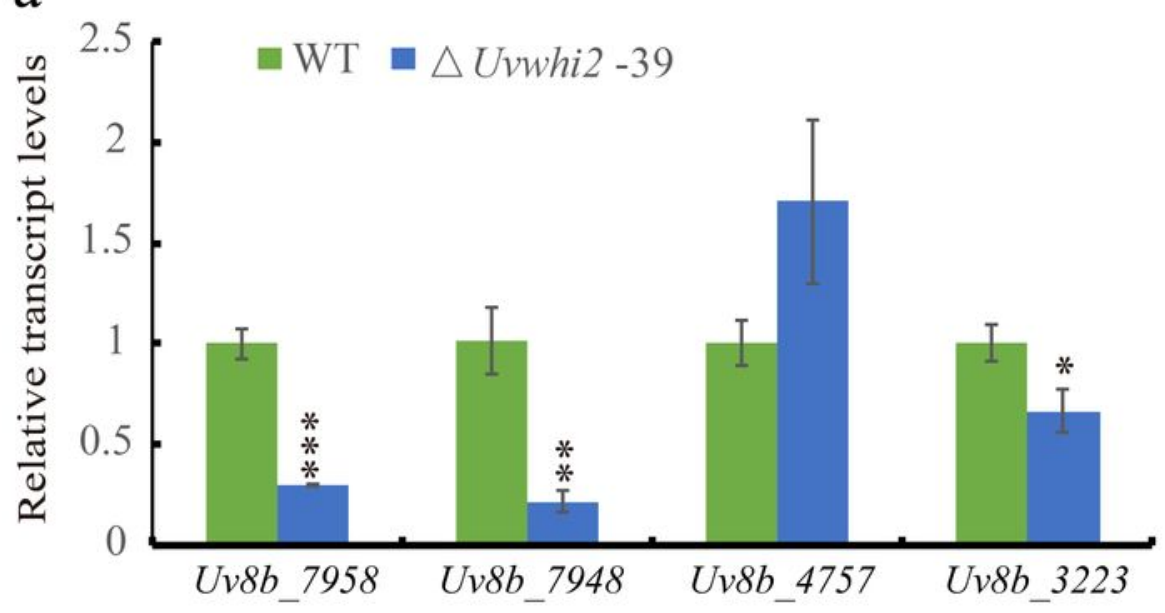

b

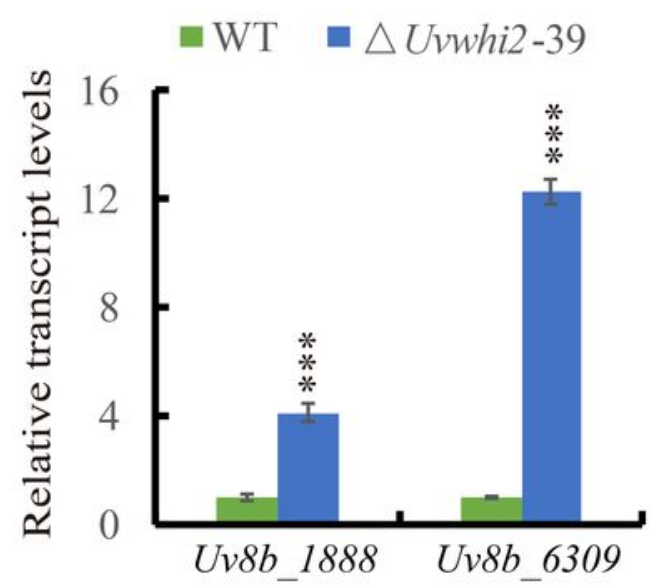

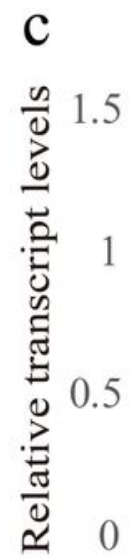

$v^{38} 8^{18^{4}} \cdot a^{9}$

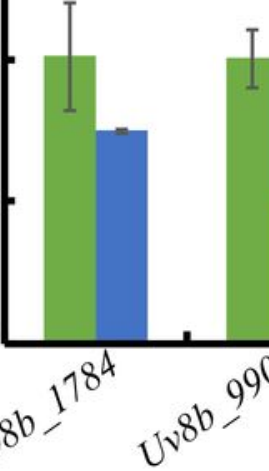

घ WT $\triangle U v w h i 2-39$
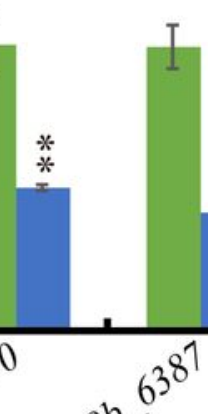

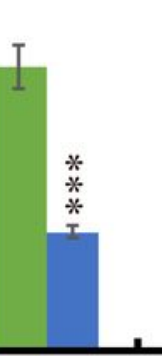

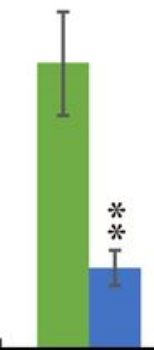

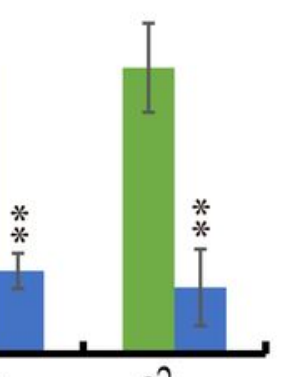
$18^{2}$

e

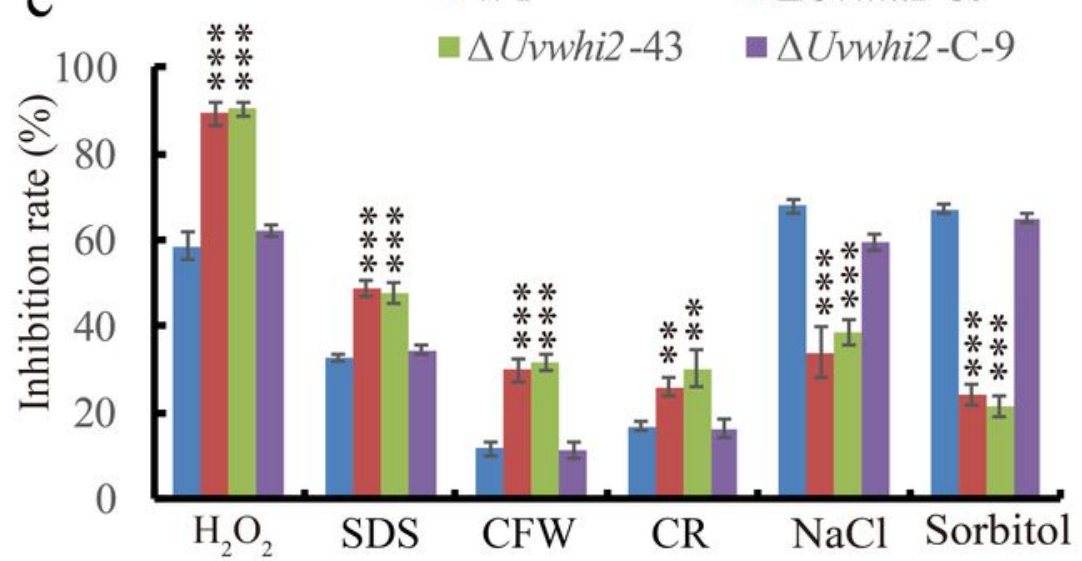

d

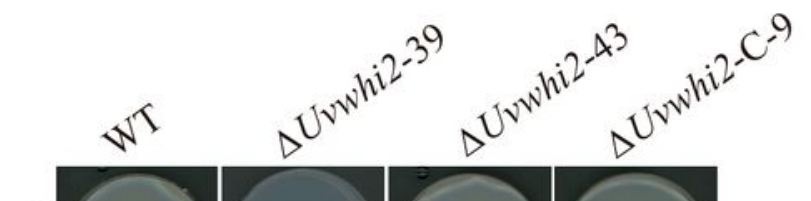


28囚. e, Statistical analysis of inhibition rate of tested strains with different stress agents. The diameters of colonies were measured and calculated. Similar results were obtained by three repeated experiments. The error bars represent the standard deviation and the asterisk represents the significant difference compared to the WT strain under the same conditions ( $\left.{ }^{*}, \mathrm{p}<0.01 ; * *, \mathrm{p}<0.005 ; * * *, p<0.001\right)$.
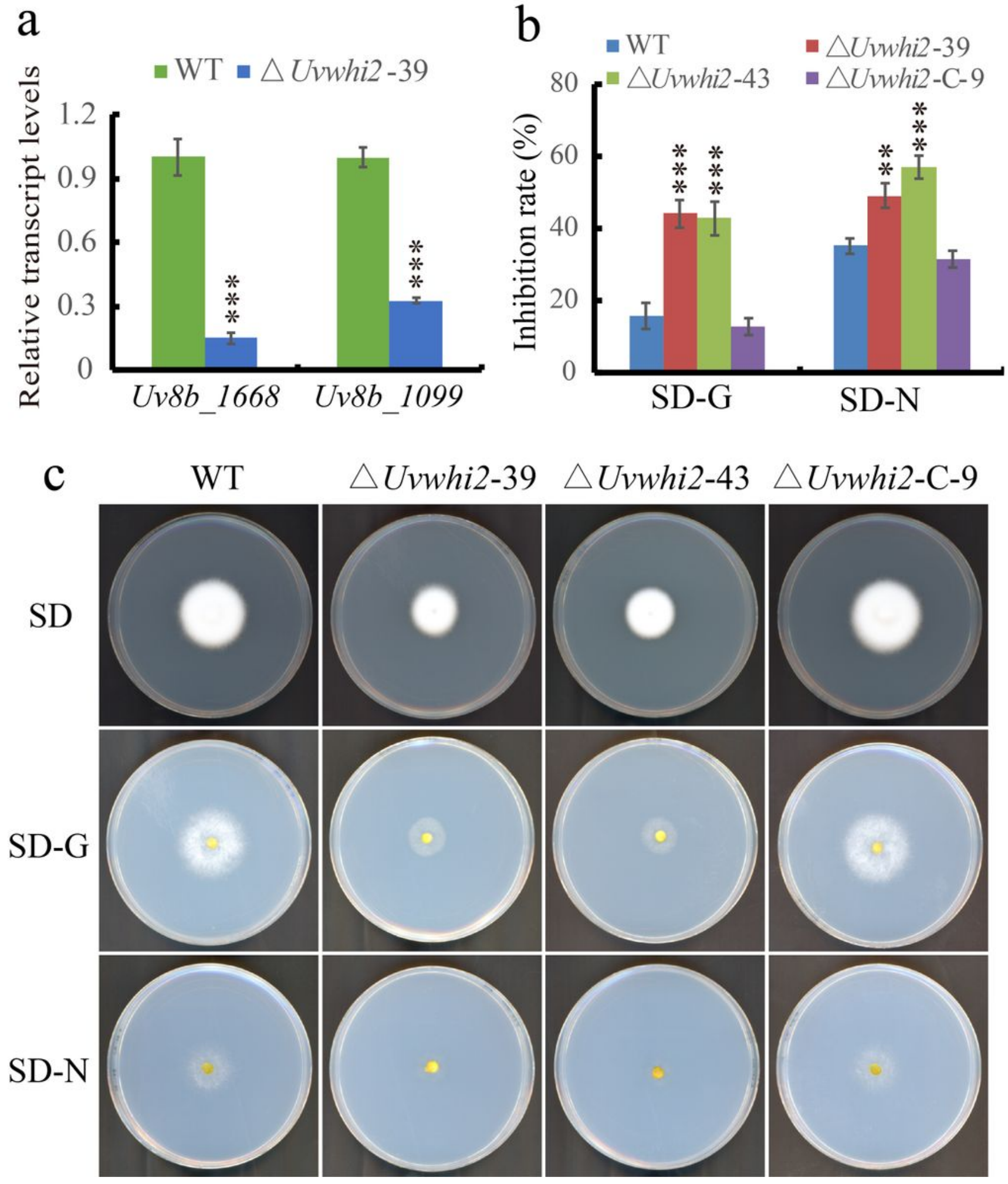

Figure 6 
UvWhi2 is involved in the regulation of nutrient stresses responses in U. virens. a, qRT-PCR analysis of the genes related to sugar synthesis. b, The growth of tested strains on the SD (synthetic dropout medium), SD-G (synthetic dropout medium without glucose), and SD-N (synthetic dropout medium without nitrogen) medium. Typical cultures were photographed after culturing for $15 \mathrm{~d}$ at 28囚. The diameters of colonies were measured to calculate the inhibition rate. c, Statistical analysis of tested strains grown on the SD-G and SD-N medium at $15 \mathrm{~d}$. Similar results were obtained by three repeated experiments. ** or $\star * *, p<0.005$ or $p<0.001$.

a

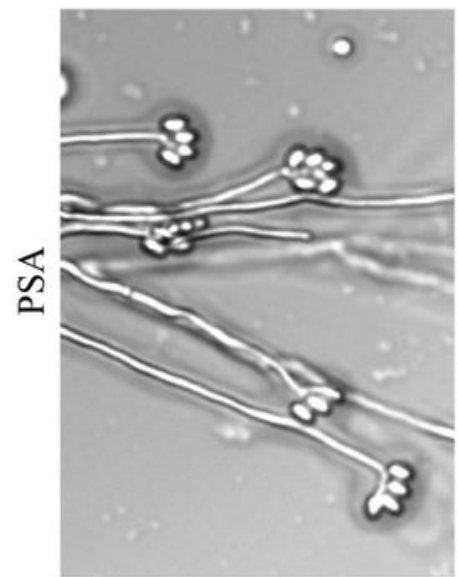

$\mathrm{b}$

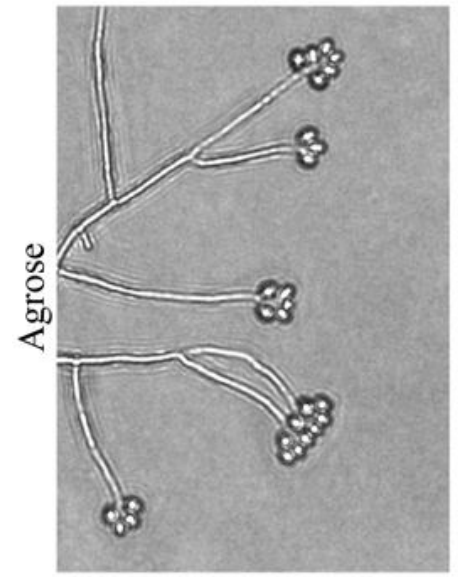

$\mathrm{c}$

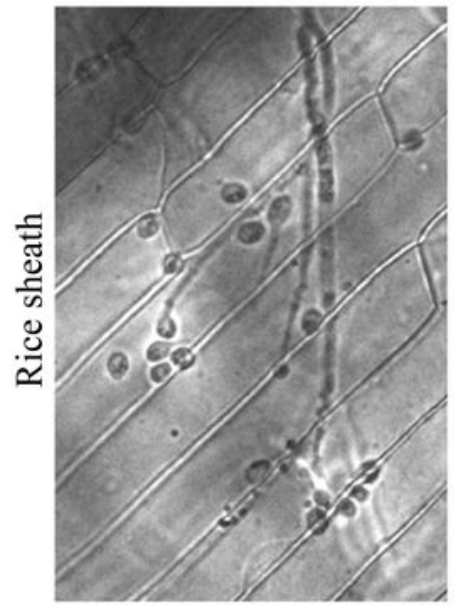

$\triangle$ UvWhi2-39

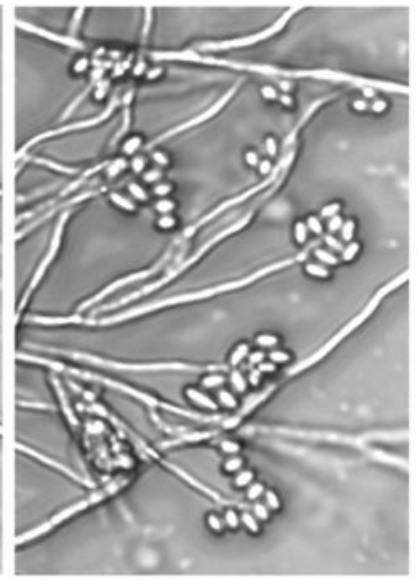

$\triangle U v W h i 2-39$

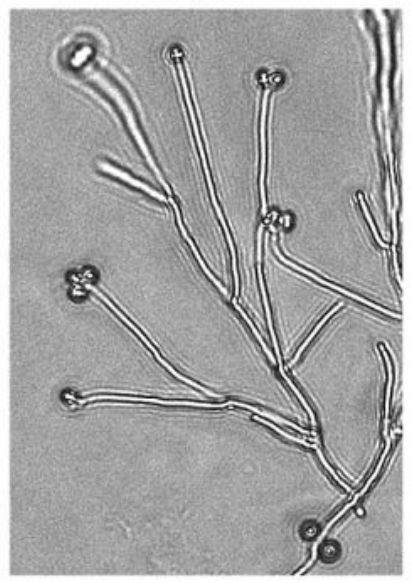

$\triangle U v W h i 2-39$

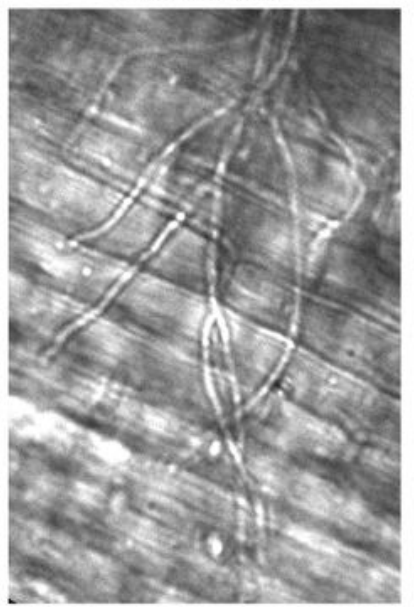

$\triangle U v W h i 2-43$

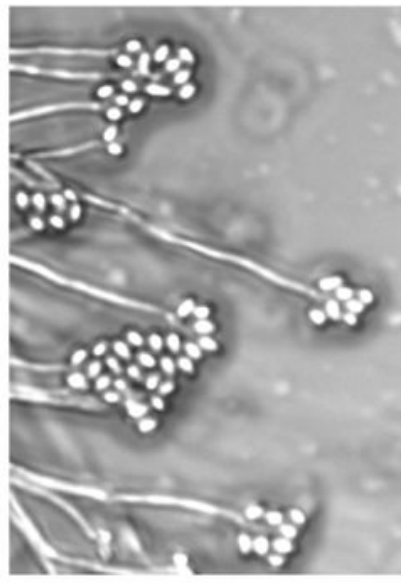

$\triangle U v W h i 2-43$

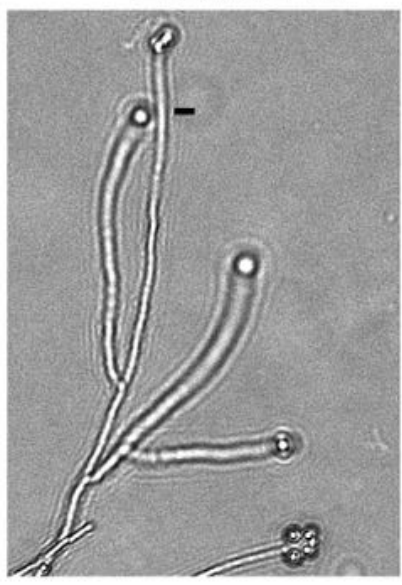

$\triangle U v W h i 2-43$

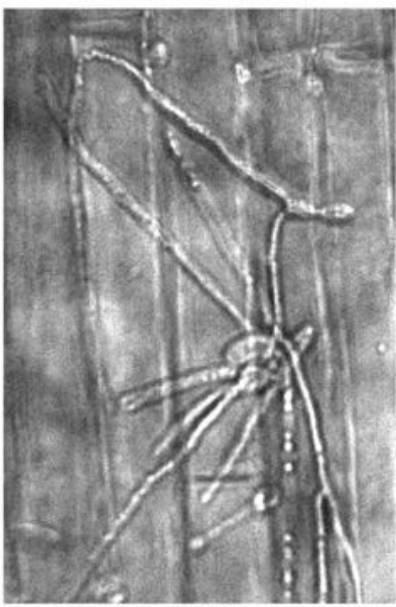

$\triangle$ UvWhi2-C-9

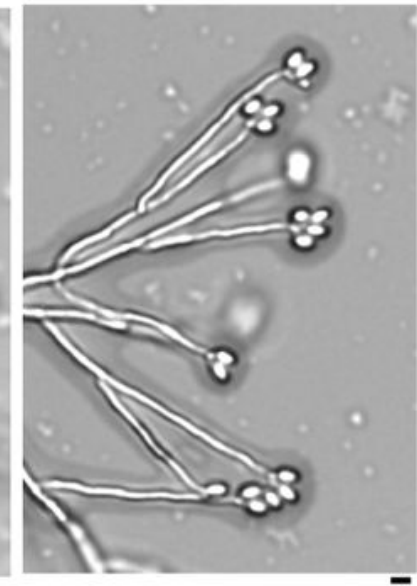

$\triangle U v W h i 2-\mathrm{C}-9$

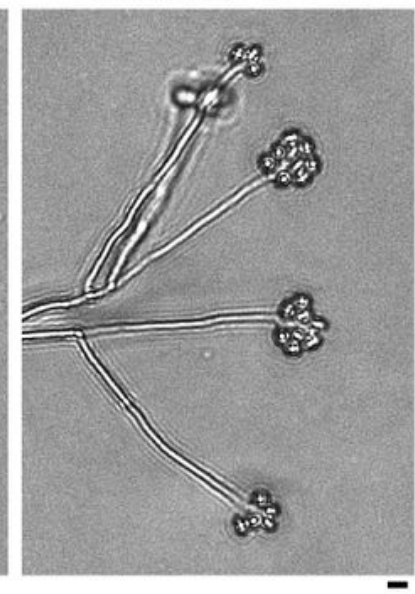

$\triangle U v W h i 2-\mathrm{C}-9$

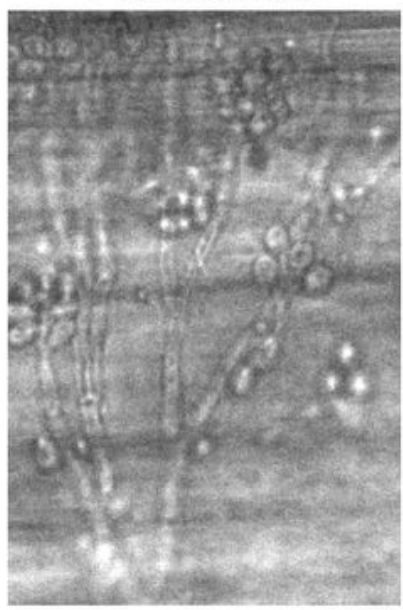




\section{Figure 7}

Conidial germination of the $\Delta$ Uvwhi2 mutant on the nutrient limited surface and rice sheath surface. a-c, Conidial germination of $U$. virens on the PSA and agarose plates and the rice sheath. The conidia were inoculated on the indicated surface at $28{ }^{\circ} \mathrm{C}$ for $3 \mathrm{~d}$. Scale bar $=3 \mu \mathrm{m}$.

\section{Supplementary Files}

This is a list of supplementary files associated with this preprint. Click to download.

- TableS1.doc

- TableS1.doc

- TableS1.doc

- TableS2.xlsx

- Tables2.xIsx

- Tables2.xlsx

- FigS1.pptx

- Figs1.pptx

- Figs1.pptx 